\title{
A Process Associated with the Radially Symmetric Dirac Equation
}

By

\author{
Brian JEFFERIES*
}

\begin{abstract}
The dynamical group associated with the Dirac equation with a radially symmetric potential is represented in terms of integrals with respect to the operator valued set functions associated with the Dirac equation in four space-time dimensions.
\end{abstract}

\section{Introduction}

It has been suggested that the Dirac equation is only of historical interest for contemporary quantum physics. Nevertheless, V.S. Popov [P1, P2] has argued that the Dirac equation with a Coulomb potential provides a good model for the spectra of one electron atoms with critical nuclear charge, in whose regime quantum electrodynamic effects are negligible. Questions concerning the essential selfadjointness of Dirac operators seem best to be answered by the introduction of the physically relevant term corresponding to an anomalous magnetic moment $[\mathrm{Be}]$. The present work is concerned with the path integral representation of the dynamical group associated with a radially symmetric Dirac operator. The mathematical phenomenon of singular perturbations present in quantum field theory is already illustrated by the Dirac equation with a Coulomb potential, so this is a good area to test path integration techniquesthis aspect is discussed in greater detail below.

In a series of papers by $\mathrm{T}$. Ichinose and $\mathrm{H}$. Tamura [I1], [I2], [I-T1], [IT2], [I-T3], the existence and support properties of a countably additive matrix valued path-space measure for the Dirac equation in two space-time dimensions are established. The properties required of a semigroup $S$ and a spectral measure $Q$ in order that for each $t>0$, there be an associated countably additive operator valued measure $M_{t}$ were given in the article [J3], and the operator valued measure associated with the Dirac equation in two space-time dimensions

Communicated by H. Araki, June 7, 1993.

1991 Mathematics Subject Classifications: Primary: 81S40, 47D06, Secondary: 28B05, $28 \mathrm{C} 20,35 \mathrm{~L} 45$.

* School of Mathematics. The University of New South Wales, P. O. Box 1 Kensington, N.S. W. 2032, Australia. 
realised as an example of the general scheme outlined there. In four spacetime dimensions, the operator valued set functions $M_{t}$ associated with the Dirac equation are unbounded [I2], [Z], [J4].

By representing the Dirac operator in four space-time dimensions as a direct sum of operators unitarily equivalent to operators acting on a space of $\mathbb{C}^{2}$-valued functions on the positive real line, T. Ichinose and H. Tamura [I-T2] also constructed families of matrix valued path space measures, but only for short times and restricted initial data.

The purpose of the present note is to show that the notion of integration with respect to a closable family of set functions [J1] may be applied to the same representation of the Dirac operator in order to represent the dynamical group $U(t)$ associated with the Dirac equation with a radially symmetric potential as an integral with respect to the operator valued set function $M_{c}$ for each $t>0$. The restriction to radially symmetric potentials is related to the well-known fact that a symmetric hyperbolic system is $L^{p}$-bounded for $p \neq 2$ if and only if the associated system of hermitian matrices commutes $[\mathrm{Br}]$; this excludes the Dirac operator. The radial symmetry realises the Dirac operator as a sum of operators unitarily equivalent to hyperbolic systems with a single matrix coefficient of the radial derivative, at the expense of introducing a $1 / r$ singularity at zero. The radial symmetry thereby facilitates control of convergence in the path integral.

The term "process" mentioned in the title refers to the underlying random process in which the operator valued set functions $M_{t}, t>0$ are used to measure random events in place of a probability measure. The idea of associating set functions with a general semigroup $S$ and spectral measure $Q$ is due to I. Kluvánek [Klu].

Ideas similar to those of [J3, Theorem 3] and [I-T3] establish the property that $M_{t}$ is closable with respect to a family of operator valued measures $M_{t}^{(s)}, \varepsilon>0$ supported on the space $\Omega$ of radial paths in $\mathbb{R}^{3}$ with speed of light c. If $V$ is a suitable radially symmetric potential, then the dynamical group $U$ associated with the Dirac equation has the representation

$$
U(t)=\int_{\Omega} \exp \left[-i \int_{0}^{t} V(\omega(s)) d s\right] d M_{t}(\omega)
$$

for all $t>0$, in the fashion of the Feynman-Kac formula for the Wiener process.

An unfortunate consequence of the support property of the operator valued measures $M_{t}^{(\varepsilon)}, \varepsilon>0$ is that for the Coulomb potentials defined for each $a>0$ by $x \rightarrow V_{a}(x)=-a /|x|, x \in \mathbb{R}^{3} \backslash\{0\}$, the function $s \mapsto V_{a}(\omega(s)), 0 \leqq s \leqq t$ is not integrable on $[0, t]$ for a set of paths $\omega \in \Omega$ which is non-null with respect to the operator valued measure $M_{t}^{(\varepsilon)}, \varepsilon>0$; these paths $\omega$ first hit the origin at some time $0<t_{\omega}<t$, so that the integral of the function $s \mapsto V_{a}(\omega(s))$ has a logarithmic singularity in a neighbourhood of $t_{\omega}$. For the Dirac operator, it is 
well-known that the Coulomb potential is singular in the sense that squaring the Dirac equation gives rise to a singularity more like $k / r^{2}$ in the Schrödinger equation-it turns out that only for $a \leqq \sqrt{3} / 2$ is the Dirac operator with the potential $V_{a}$ essentially selfadjoint on $C_{c}^{\infty}\left(\mathbb{R}^{3} \backslash\{0\} ; \mathbb{C}^{+}\right)[\mathrm{W}$, Theorem 6.9]. In the range $\sqrt{ } 3 / 2<a<1$ there is a "physical" selfadjoint extension which ceases to exist in the range $a \geqq 1$ (see $[\mathrm{KS}]$ and the references there).

The situation for the Dirac equation is in contrast with the Schrödinger equation in $\mathbb{R}^{3}$. There the Feynman-Kac formula for the Wiener process picks out the "physical" selfadjoint extension of $-1 / 2 \Delta+V$ in the case that the form sum of the free Hamiltonian $-1 / 2 \Delta$ and the potential $V$ is bounded below. The potential $V$ may have singularities on a set of capacity zero.

The natural interpretation in the present context is that the Kac functional $\omega \mapsto \exp \left[-i \int_{0}^{t} V(\omega(s)) d s\right], \omega \in \Omega$ is not the right multiplicative functional to use in the case of the Coulomb potential or other singular potentials associated with physically reasonable dynamics. The situation is reflected in the $P(\phi)_{2}$ Euclidean quantum field theory [G-J], where the construction of multiplicative functionals of the field involves "renormalisation".

General results guaranteeing the existence of bounded $(S, Q, t)$-set functions on $L^{p}$-spaces are presented in section one. Properties of the semigroups associated with the Dirac operator $D$ are established in section two. Here a number of results concerning approximation of semigroups to be used in section six are established.

As mentioned previously, the free Dirac operator $D$ with mass $m \in \mathbb{R}$ may be represented as the direct sum of operators unitarily equivalent to the differential operators

$$
\tau_{k}: g \longmapsto\left(\begin{array}{cc}
0 & -1 \\
1 & 0
\end{array}\right)\left(\begin{array}{l}
g_{1}{ }^{\prime}(r) \\
g_{2}{ }^{\prime}(r)
\end{array}\right)+\left(\begin{array}{cc}
m & -k r^{-1} \\
-k r^{-1} & -m
\end{array}\right)\left(\begin{array}{l}
g_{1}(r) \\
g_{2}(r)
\end{array}\right), \quad g \in C_{c}^{\infty}\left((0, \infty) ; \mathbb{C}^{2}\right),
$$

for $k \in \mathbb{Z} \backslash\{0\}$. The operators $\tau_{k}$ are essentially selfadjoint [W, Theorem 6.9], so we actually need to use their closures in $L^{2}\left((0, \infty) ; \mathbb{C}^{2}\right)$. For each $\varepsilon>0, M_{t}^{(\varepsilon)}$ is the operator valued set function associated with the family of operators $(0.1)$ in which the expression $k r^{-1}$ is replaced by $k r^{-1}$ for $r>\varepsilon$ and by $k \varepsilon^{-1}$ for $r \leqq \varepsilon$. The results of section one ensure that $M_{t}^{(s)}, \varepsilon>0$ are operator valued measures. The diameter of the range of $M_{t}^{(s)}, \varepsilon>0$ diverges as $\varepsilon \rightarrow 0^{+}$.

In section four we establish the support property of $M_{t}^{(\varepsilon)}, t>0$ by writing $M_{t}^{(\varepsilon)}$ in terms of a perturbation expansion. Section five is devoted to proving, in the terminology of $[\mathrm{J} 1]$, that for each $t>0$, the $\left(S_{D}, Q_{\mathrm{R}}, t\right)$-set function, for the dynamical group $S_{D}$ associated with the free Dirac equation in four spacetime dimensions and the spectral measure $Q_{\mathrm{R}}$ of multiplication by radially symmetric Borel subsets of $\mathbb{R}^{3}$ is closable with respect to $M_{t}^{(s)}, \varepsilon>0$. Integration with respect to $M_{t}$ is also supported by $\Omega$. 
Thus, armed with the notion of integration with respect to $M_{t}$ relative to $M_{t}^{(s)}, \varepsilon>0$, the representation of the dynamical group $U(t), t>0$ associated with the Dirac equation with a suitable spherically symmetric potential, in terms of an integral with respect to $M_{t}$, is established in section six, see Theorem 6.3.

The existence of the dynamical group $U$ is most easily verified by traditional operator theoretic means. However, as noted above, the Dirac equation shares features with Euclidean quantum field theory in two and three space-time dimensions, where there is a corpus of ad hoc techniques for the construction of dynamical groups. The systematic use of real time functional integration may reveal some new mathematical structure underlying the current proofs of the existence of non-trivial dynamics in two and three space-time dimensions. Already for the Dirac equation, the need to use some multiplicative functional other than the Kac functional $\omega \mapsto \exp \left[-i \int_{0}^{t} V(\omega(s)) d s\right], \omega \in \Omega$ to represent the dynamical group for the Coulomb potential $V$ is suggestive of the situation in Euclidean quantum field theory.

\section{$\S 1$. $(S, Q, t)$-Measures}

Some facts concerning $(S, Q, t)$-set functions for semigroups acting on $L^{p}$ spaces are collected in this section. First we fix some notation.

All vector space are assumed to be over the complex scalars. A locally convex space $X$ is said to be quasi-complete if every closed and bounded subset is complete. In particular, a quasi-complete space is sequentially complete. The space of all continuous linear operators on a locally convex space $X$ is denoted by $\mathcal{L}(X)$. It is endowed with the topology of strong convergence. If $X$ is a Banach space, then by the uniform boundedness principle, $\mathcal{L}(X)$ is quasicomplete.

The identity operator on $X$ is denoted by $I$. A semigroup $S$ of operators is a function $S:[0, \infty) \rightarrow \mathcal{L}(X)$ such that $S(t) S(s)=S(t+s)$ for all $s, t \geqq 0$. A semigroup $S$ is called a $C_{0}$-semigroup if $\lim _{t \rightarrow 0}+S(t)=I$ in $\mathcal{L}(X)$; this terminology applies exclusively to a Banach space $X$ in this work.

At some stages in the arguments of the present paper, it is necessary to integrate vector valued functions with respect to vector or operator valued measures. Fortunately, only the finite dimensional case is needed, so certain technical difficulties are avoided. For example, if $T: \mathcal{L}\left(\mathbb{C}^{n}\right) \times \mathcal{L}\left(\mathbb{C}^{n}\right) \rightarrow \mathcal{L}\left(\mathbb{C}^{n}\right)$ is a norm continuous bilinear map such as $(A, B) \mapsto A B, A \in \mathcal{L}\left(\mathbb{C}^{n}\right), B \in \mathcal{L}\left(\mathbb{C}^{n}\right)$, then Bartle's bilinear integral $[B]$ is sufficient for our purposes. Another case we need is for the example of the bilinear map $(A, x) \mapsto A x, A \in \mathcal{L}\left(\mathbb{C}^{n}\right), x \in \mathbb{C}^{n}$.

The variation of a scalar valued set function $m$ is (an extended-real valued set function) denoted by $|m|$. The set $[0, \infty)$ is denoted by $\mathbb{R}_{+}$. The space of all smooth $\mathbb{C}^{2}$-valued functions on $(0, \infty)$ with compact support is denoted 
by $C_{c}^{\infty}\left((0, \infty), \mathbb{C}^{2}\right)$. The Borel $\sigma$-algebra of a Hausdorff topological space $\Sigma$ is denoted by $\mathscr{B}(\Sigma)$.

Let $(\Sigma, \mathscr{I}, \mu)$ be a $\sigma$-finite measure space and let $X$ be a Banach space with norm denoted by $\|\cdot\|_{X}$. For each $1 \leqq p<\infty,\left(L^{p}(\mu ; X),\|\cdot\|_{p}\right)$ denotes the Banach space of ( $\mu$-equivalence classes of) strongly $\mu$-measurable functions $f$ : $\Sigma \rightarrow X$ such that $\|f\|_{p} \equiv\left(\int_{\Sigma}\|f(\sigma)\|_{X}^{p} d \mu(\sigma)\right)^{1 / p}$ is finite. For $p=\infty,\left(L^{\infty}(\mu ; X),\|\cdot\|_{\infty}\right)$ denotes the Banach space of ( $\mu$-equivalence classes of) strongly $\mu$-measurable functions $f: \Sigma \rightarrow X$ such that the number $\|f\|_{\infty} \equiv \inf \left\{\lambda:\|f(\sigma)\|_{X} \leqq \lambda\right.$ for $\mu$-almost all $\sigma \in \Sigma\}$ is finite. For $p=2$ and $X$ a Hilbert space, $L^{2}(\mu ; X)$ is a Hilbert space with the inner product $(f, g)=\int_{\Sigma}(f(\sigma), g(\sigma)) d \mu(\sigma)$, for all $f, g \in L^{2}(\mu ; X)$. For the applications of section two, $X$ is either $\mathbb{C}^{2}$ or $\mathbb{C}^{4}$ with a $p$-norm, $1 \leqq$ $p \leqq \infty$.

Let $\mathscr{I}_{Q}$ be a sub- $\sigma$-algebra of $\mathscr{I}$ and suppose that $Q$ is the spectral measure of multiplication by characteristic functions of sets belonging to $\mathscr{I}_{Q}$. Then for each $A \in I_{Q}, Q(A)$ defines a bounded linear operator on each space $L^{p}(\mu), 1 \leqq$ $p \leqq \infty$. The same symbol denotes the operator acting on each of these spaces. Let $\Sigma_{Q}$ be a set for which $Q\left(A \cap\left(\Sigma \backslash \Sigma_{Q}\right)\right)=0$ for all $A \in I_{Q}$. In the case that $Q$ is regular, $\Sigma_{Q}$ is assumed to be its support.

Now suppose that $i A$ is the generator of a $\mathrm{C}_{0}$-semigroup acting on the Hilbert space $L^{2}(\mu)$. For example, if $A$ is selfadjoint, then $S_{A}(t)=e^{i A t}, t \in \mathbb{R}$ is a unitary group of operators, by Stone's theorem [R-S, VIII.8]. However, in the examples of interest in section two, $A$ is not selfadjoint. Let $t>0$ be fixed.

Denote by $\Omega_{\infty}$ the collection of all functions $\omega:[0, \infty) \rightarrow \Sigma_{Q}$. For any set

$$
E=\left\{\boldsymbol{\omega} \in \Omega_{\infty}: \boldsymbol{\omega}\left(t_{1}\right) \in B_{1}, \cdots, \boldsymbol{\omega}\left(t_{n}\right) \in B_{n}\right\}
$$

with $0<t_{1}<\cdots<t_{n}<t$, and with $B_{1}, \cdots, B_{n}$ belonging to $\mathscr{I}_{Q}$, the operators

$$
\left\{\begin{array}{l}
M_{A, t}(E)=S_{A}\left(t-t_{n}\right) Q\left(B_{n}\right) S_{A}\left(t_{n}-t_{n-1}\right) \cdots S_{A}\left(t_{2}-t_{1}\right) Q\left(B_{1}\right) S_{A}\left(t_{1}\right) \\
M_{A, t} \#(E)=S_{A}\left(t_{1}\right) Q\left(B_{1}\right) S_{A}\left(t_{2}-t_{1}\right) \cdots S_{A}\left(t_{n}-t_{n-1}\right) Q\left(B_{n}\right) S_{A}\left(t-t_{n}\right)
\end{array}\right.
$$

act on the Hilbert space $L^{2}(\mu)$.

The collection of all such sets $E$ as $0<t_{1}<\cdots<t_{n}<t, B_{1}, \cdots, B_{n}$ and $n=1$, $2, \cdots$ vary is denoted by $\mathcal{S}_{t}$. Then $\mathcal{S}_{t}$ is a semi-algebra of subsets of the set $\Omega_{\infty}$. The suggestive terminology is that $\mathcal{S}_{t}$ is the collection of elementary events before time $t$. Sometimes sets in the algebra $\alpha\left(\mathcal{S}_{t}\right)$ generated by $\mathcal{S}_{t}$ will be referred to as cylinder sets.

The semigroup and spectral measure properties ensure that $M_{A, t}, M_{A, t} \#$ are additive operator valued set functions on $\mathcal{S}_{t}$. It follows that $M_{A, t}, M_{A, t}{ }^{\#}$ have unique additive extensions to the algebra $\alpha\left(\mathcal{S}_{t}\right)$ generated by $\mathcal{S}_{t}$. We denote the extension by the same symbol. The set function $M_{A, t}$ is called the $\left(S_{A}, Q, t\right)$ set function. 
Our next aim is to give a condition for which the $\mathcal{L}\left(L^{2}(\mu)\right)$-valued set functions $M_{A, t}: \alpha\left(\mathcal{S}_{t}\right) \rightarrow \mathcal{L}\left(L^{2}(\mu)\right), M_{A, t} \#: \alpha\left(\mathcal{S}_{t}\right) \rightarrow \mathcal{L}\left(L^{2}(\mu)\right)$ have bounded ranges, in which case they are said to be bounded.

1.1 Lemma. Suppose that $i A$ is the generator of a contraction $\mathrm{C}_{0}$-semigroup acting on the Hilbert space $L^{2}(\mu)$ such that for each $f \in L^{1}(\mu) \cap L^{2}(\mu)$, the inequalities $\left\|e^{\imath A t} f\right\|_{1} \leqq\|f\|_{1}$ and $\left\|\left(e^{\imath A t}\right)^{*} f\right\|_{1} \leqq\|f\|_{1}$ hold for all $t \geqq 0$.

Then for each $1 \leqq p<\infty$, there exists a unique linear operator $A_{p}: \mathscr{D}\left(A_{p}\right) \rightarrow$ $L^{p}(\mu)$ such that $i A_{p}$ is the infinitesimal generator of a $\mathrm{C}_{0}$-contraction semigroup $T_{p}$ on $L^{p}(\mu)$ and $T_{p}(t) f=e^{2 A t} f$ for all $f \in L^{p}(\mu) \cap L^{2}(\mu)$ and all $t \geqq 0$.

For $p=\infty$, there exists a unique linear operator $A_{\infty}: \mathscr{D}\left(A_{\infty}\right) \rightarrow L^{\infty}(\mu)$ such that $i A_{\infty}$ is the infinitesimal generator of a weak*-continuous contraction semigroup $T_{\infty}$ on $L^{\infty}(\mu)$ and $T_{\infty}(t) f=e^{\imath \Lambda t} f$ for all $f \in L^{\infty}(\mu) \cap L^{2}(\mu)$ and all $t \geqq 0$.

Proof. We first make a few obvious remarks. If $S(t), t \geqq 0$ is a family of bounded linear operators on $X$ such that for some dense set of vectors $x \in X$, the equality $S(t) S(s) x=S(t+s) x$ holds for all $s, t \geqq 0$, then $S$ is actually a semigroup of operators. If, in addition, $S(t), t>0$ is uniformly bounded in a neighbourhood of $t=0$ and $\lim _{t \rightarrow 0} S(t) x=x$ for a dense set of vectors $x \in X$, then $S$ is a $\mathrm{C}_{0}$-semigroup of operators acting on $X$.

Because $L^{1}(\mu) \cap L^{2}(\mu)$ is dense in $L^{1}(\mu)$, for each $t \geqq 0$, there exist unique operators $T_{1}(t): L^{1}(\mu) \rightarrow L^{1}(\mu), S_{1}(t): L^{1}(\mu) \rightarrow L^{1}(\mu)$ such that $T_{1}(t) f=e^{i A t} f$ and $S_{1}(t) f=\left(e^{i A t}\right) * f$ for all $f \in L^{1}(\mu) \cap L^{2}(\mu)$. Now the measure $\mu$ is not assumed to be finite, so the space $L^{1}(\mu) \cap L^{\infty}(\mu) \cap L^{2}(\mu)$ need not be dense in $L^{\infty}(\mu)$. Consequently, an alternate argument is needed for the case of $L^{\infty}(\mu)$.

The duality between $L^{1}(\mu)$ and $L^{\infty}(\mu)$ is given by $\langle f, g\rangle=\int_{\Sigma} f(\sigma) g(\sigma) d \mu(\sigma)$, $f \in L^{1}(\mu), g \in L^{\infty}(\mu)$. For $f \in L^{1}(\mu) \cap L^{2}(\mu), g \in L^{\infty}(\mu) \cap L^{2}(\mu)$, the inner product of $f$ with $g$ is $(f, g)=\int_{\Sigma} f(\sigma) \overline{g(\sigma)} d \mu(\sigma)$, so $\langle f, g\rangle=(f, J g)$ for the antilinear complex conjugation operator $J: L^{2}(\mu) \rightarrow L^{2}(\mu)$. Furthermore, $\left\langle S_{1}(t) f, g\right\rangle=$ $\left\langle\left(e^{i A t}\right)^{*} f, g\right\rangle=\left(\left(e^{i A t}\right)^{*} f, J g\right)=\left(f, e^{i \Lambda t} J g\right)=\left\langle f, J e^{i A t} J g\right\rangle$, so the Banach space dual operator of $S_{1}(t): L^{1}(\mu) \rightarrow L^{1}(\mu)$ is $J e^{i A t} J$.

However, for any $g \in L^{\infty}(\mu) \cap L^{2}(\mu)$, there exists $g_{n} \in L^{1}(\mu) \cap L^{\infty}(\mu) \cap L^{2}(\mu)$, $n=1,2, \cdots$ such that $\left\|g_{n}\right\|_{\infty} \leqq\|g\|_{\infty}$ for all $n=1,2, \cdots$ and $g_{n} \rightarrow g$ in $L^{2}(\mu)$ and $\mu$ almost everywhere. Such a sequence may be obtained, for example, from the martingale convergence theorem. Then for every $f \in L^{1}(\mu) \cap L^{2}(\mu),\left\langle f, J e^{i A t} g_{n}\right\rangle$ $=\left\langle S_{1}(t) f, J g_{n}\right\rangle \rightarrow\left\langle S_{1}(t) f, J g\right\rangle$ by dominated convergence, and $\left\langle f, J e^{\imath A t} g_{n}\right\rangle \rightarrow$ $\left\langle f, J e^{i A t} g\right\rangle$ by convergence in $L^{2}(\mu)$. If follows that $\left|\left\langle f, J e^{i A t} g\right\rangle\right|=\left|\left\langle S_{1}(t) f, J g\right\rangle\right|$ $\leqq\|f\|_{1}\|J g\|_{\infty}$ for all $f \in L^{1}(\mu) \cap L^{2}(\mu)$. Because $L^{1}(\mu) \cap L^{2}(\mu)$ is dense in $L^{1}(\mu)$ and $J$ is norm preserving, it follows that $\left\|e^{\imath A t} g\right\|_{\infty} \leqq\|g\|_{\infty}$ for all $g \in L^{\infty}(\mu) \cap L^{2}(\mu)$.

The Riesz-Thorin interpolation theorem [R-S, Vol. II, pp 38-39] shows that 
for each $t \geqq 0$ and $1 \leqq p<\infty$, there exists a unique bounded linear operator $T_{p}(t)$ such that $\left\|T_{p}(t) f\right\|_{p} \leqq\|f\|_{p}$ for all $f \in L^{p}(\mu)$ and the equality $T_{p}(t) f=e^{i A t} f$ holds for all $f \in L^{p}(\mu) \cap L^{2}(\mu)$. The remarks at the beginning of the proof serve to show that $T_{p}$ is actually a $\mathrm{C}_{0}$-semigroup acting on $L^{p}(\mu)$. If $i A_{p}$ is its infinitesimal generator, then the operator $A_{p}$ has the required properties.

For $p=\infty$, the Banach space dual operator of $J S_{1}(t) J, t \geqq 0$ defines a weak*continuous semigroup $T_{\infty}(t), t \geqq 0$ on $L^{\infty}(\mu)$ such that $T_{\infty}(t) f=e^{i A t} f$ for all $f \in$ $L^{\infty}(\mu) \cap L^{2}(\mu)$.

Suppose that $A$ satisfies the condition of Lemma 1.1. Then $S_{A}$ defines a unique continuous semigroup of operators on $L^{p}(\mu)$ for each $1 \leqq p \leqq \infty$. Because $Q$ is a spectral measure acting on $L^{p}(\mu)$ (for $p=\infty, L^{\infty}(\mu)$ has the weak ${ }^{*}$ topology $\left.\sigma\left(L^{\infty}(\mu), L^{1}(\mu)\right)\right)$ the operators defined in (1.2) also act on $L^{p}(\mu)$ for each $1 \leqq p \leqq \infty$. The same symbols are used to denote the corresponding operators acting on each of the spaces $L^{p}(\mu), 1 \leqq p \leqq \infty$.

1.2 Lemma. Suppose that A satisfies the condition of Lemma 1.1. Then the additive set functions $M_{A, t}, M_{A, t} t^{\#}$ are bounded in $\mathcal{L}\left(L^{\infty}(\mu)\right)$ on the algebra $\alpha\left(\mathcal{S}_{t}\right)$ generated by $\mathcal{S}_{t}$. Moreover,

$$
\sup \left\{\left\|M_{A, t}(E)\right\|_{\mathcal{L}\left(L^{\infty}(\mu)\right)}: E \in \alpha\left(\mathcal{S}_{t}\right)\right\} \leqq 1, \quad \sup \left\{\left\|M_{A, t} \#(E)\right\|_{\mathcal{L}\left(L^{\infty}(\mu)\right)}: E \in \alpha\left(\mathcal{S}_{t}\right)\right\} \leqq 1
$$

for each $t>0$.

Proof. Because $S_{A}$ is a contraction on $L^{\infty}(\mu)$, the result follows from [J3, Proposition 1]. However, we distill the essential argument here for the additive set function $M_{A, t}$.

Discarding $\mu$-null sets, if necessary, for any element $E$ of the algebra $\alpha\left(\mathcal{S}_{t}\right)$, the additivity of $M_{A, t}$ ensures that operator $M_{A, t}(E)$ may be represented as the sum of the operators $M_{A, t}\left(E_{k, \jmath}\right)$ with

$$
E_{k, j}=\left\{\omega \in \Omega: \omega\left(t_{1}\right) \in B_{1}^{k, j}, \cdots, \omega\left(t_{n-1}\right) \in B_{n-1}^{k, j}, \omega\left(t_{n}\right) \in B_{n}^{k}\right\}
$$

$j=1, \cdots, J(k), k=1, \cdots, m$ for $0<t_{1}<\cdots<t_{n}<t$. The sets $B_{n}^{k} \in \mathscr{F}_{Q}, k=1, \cdots, m$ are assumed to be pairwise disjoint. Then

$$
M_{A, t}(E)=\sum_{k=1}^{m} M_{A, t}\left(\bigcup_{j=1}^{J(k)} E_{k, j}\right)=\sum_{k=1}^{m} S_{A}\left(t-t_{n}\right) Q\left(B_{n}^{k}\right) M_{A, t_{n}}\left(\bigcup_{j=1}^{J(k)} F_{k, j}\right)
$$

with $F_{k, j}=\left\{\omega \in \Omega: \omega\left(t_{1}\right) \in B_{1}^{k, j}, \cdots, \omega\left(t_{n-1}\right) \in B_{n-1}^{k, j}\right\}$.

If we knew that the operator $T_{k}=M_{A, t_{n}}\left(\bigcup_{j=1}^{J(k)} F_{k, j}\right)$ had its norm bounded by one for each $k=1, \cdots, m$, then so would the operators $\sum_{k=1}^{m} Q\left(B_{n}^{k}\right) T_{k}$ and $\sum_{k=1}^{m} S\left(t-t_{n}\right) Q\left(B_{n}^{k}\right) T_{k}$, because for all $f_{k} \in L^{\infty}(\mu), k=1, \cdots, m$ in the closed unit ball of $L^{\infty}(\mu)$, the inequalities $\left|\sum_{k=1}^{m}\left(Q\left(B_{n}^{k}\right) f_{k}\right)(\sigma)\right| \leqq \sum_{k=1}^{m}\left|\left(Q\left(B_{n}^{k}\right) f_{k}\right)(\sigma)\right|=$ $\sum_{k=1}^{m} \chi_{B_{n}^{k}}(\sigma)\left|f_{k}(\sigma)\right| \leqq 1$ hold for $\mu$-almost all $\sigma \in \Sigma$. It is clear that the proof can now be completed by induction. The analogous argument holds for the additive 
set function $M_{A, t}$.

1.3 Proposition. Suppose that iA is the generator of a contraction $\mathrm{C}_{0}$-semigroup acting on the Hilbert space $L^{2}(\mu), N$ is a unitary operator acting on $L^{2}(\mu)$ and $K \geqq 0$ is a number such that for each $f \in L^{1}(\mu) \cap L^{2}(\mu)$, the inequalities $\left\|N^{*} e^{i A t} N f\right\|_{1}$ $\leqq e^{K t}\|f\|_{1},\left\|N\left(e^{i A t}\right) * N^{*} f\right\|_{1} \leqq e^{K t}\|f\|_{1}$ hold for all $t \geqq 0$. Suppose also that $N$ and $Q$ commute.

Then for each $1 \leqq p \leqq \infty, t>0,\left\|N^{*} M_{A, t}(E) N f\right\|_{p} \leqq e^{K t}\|f\|_{p}$ for all $f \in L^{p}(\mu) \cap$ $L^{2}(\mu)$ and all sets $E$ belonging to the algebra $\alpha\left(\mathcal{S}_{t}\right)$ of sets generated by $\mathcal{S}_{t}$.

Proof. Let $\mathscr{D}(B)=N^{*} \mathscr{D}(A)$ and set $B=N^{*} A N-K I$. Let $J$ be the complex conjugation operator on $L^{2}(\mu)$ and set $C=J B^{*} J$. Then $i B$ is the infinitesimal generator of the $\mathrm{C}_{0}$-contraction semigroup $e^{i B t}=e^{-K t} N^{*} e^{i A t} N, t \geqq 0$ satisfying the conditions of Lemma 1.2. Similarly, $i C$ is the infinitesimal generator of the $\mathrm{C}_{0}$-contraction semigroup $J\left(e^{i B t}\right) * J, t \geqq 0$ satisfying the conditions of Lemma 1.2. The adjoint semigroup $t \mapsto\left(e^{i B t}\right)^{*}, t \geqq 0$ is clearly weakly continuous on $L^{2}(\mu)$, but this implies that it is also strongly continuous too [H-P, 10.6.5].

Moreover, the equalities $\left\langle e^{i B t} f, g\right\rangle=\left(e^{i B t} f, J g\right)=\left(f, e^{-i B^{*} t} J g\right)=\left\langle f, J e^{-i B^{* t}} J g\right\rangle$ valid for all $f \in L^{1}(\mu) \cap L^{2}(\mu)$ and $g \in L^{\infty}(\mu) \cap L^{2}(\mu)$ ensure, in the notation of Lemma 1.1, that $i C_{\infty}$ is the infinitesimal generator of the weak *-continuous semigroup $e^{i C_{\infty} t}, t \geqq 0$ adjoint to $e^{i B_{1} t}, t \geqq 0$ with respect to the duality between $L^{1}(\mu)$ and $L^{\infty}(\mu)$. The semigroup $e^{i C t}, t \geqq 0$ also satisfies the conditions of Lemma 1.2. An application of Lemma 1.2 to the operators $B$ and $C$ establishes the inequalities $\sup \left\{\left\|M_{B, t}(E)\right\|_{\mathcal{L}\left(L^{\infty}(\mu)\right)}: E \in \alpha\left(\mathcal{S}_{t}\right)\right\} \leqq 1$ and $\sup \left\{\left\|M_{C, t} \#(E)\right\|_{\left.\mathcal{L}: L^{\infty}(\mu)\right)}\right.$ : $\left.E \in \alpha\left(\mathcal{S}_{t}\right)\right\} \leqq 1$. It is easily checked that for each $E \in \alpha\left(\mathcal{S}_{t}\right)$, the dual of the operator $M_{C, t} \#(E): L^{\infty}(\mu) \rightarrow L^{\infty}(\mu)$ with respect to the duality $\langle\cdot, \cdot\rangle$ between $L^{1}(\mu)$ and $L^{\infty}(\mu)$ is $M_{B, t}(E): L^{1}(\mu) \rightarrow L^{1}(\mu)$, so by duality, the norm estimate $\sup \left\{\left\|M_{B, t}(E)\right\|_{\mathcal{L}\left(L_{1}(\mu)\right)}: E \in \alpha\left(\mathcal{S}_{t}\right)\right\} \leqq 1$ holds. An application of the Riesz-Thorin interpolation theorem [R-S, Vol. II, pp 38-39] shows that $\left\|M_{B, t}(E) f\right\|_{p} \leqq\|f\|_{p}$ for all $f \in L^{p}(\mu) \cap L^{2}(\mu)$ and all sets $E$ belonging to the algebra $\alpha\left(\mathcal{S}_{t}\right)$ of sets generated by $\mathcal{S}_{t}$. According to formula (1.2), the equality $M_{B, t}(E)=$ $e^{-K t} N^{*} M_{A, t}(E) N$ holds for all sets $E \in \mathcal{S}_{t}$, because $N$ and $Q$ commute. By the additivity of the set functions $M_{B, t}$ and $M_{A, t}$, the equality must hold on the algebra $\alpha\left(\mathcal{S}_{t}\right)$ generated by $\mathcal{S}_{t}$; the conclusion follows.

Up until now, we have been concerned not with the $\sigma$-additivity of the set functions $M_{A, t}$, but only with their boundedness on the algebra $\alpha\left(\mathcal{S}_{t}\right)$. For the next statement, it is convenient to introduce a regularity assumption on the spectral measure $Q$.

Let $I_{m}$ be a sub- $\sigma$-algebra of the Borel subsets of a Hausdorff space $X$. Let $E$ be a locally convex space. A vector valued measure $m: \mathscr{T}_{m} \rightarrow E$ is said to be regular, if for every $A \in I_{m}$ and every neighbourhood $U$ of zero in $E$, 
there exists a set $B \in \mathscr{I}_{m}$ and a compact subset $K$ of $X$ such that $B \cong K \cong A$, and $m(C) \in U$ for all $C \in \mathscr{I}_{n}$ such that $C \leqq A \backslash B$.

1.4 Theorem. Let $\Sigma$ be a locally compact Hausdorff space, $\mu$ a $\sigma$-finite regular Borel measure in $\Sigma$. Let $Q$ be a regular spectral measure of multiplication by characteristic functions of a sub- $\sigma$-algebra of the Borel subsets of $\Sigma$. Suppose that $i A$ is the generator of a contraction $\mathrm{C}_{0}$-semigroup acting on the Hilbert space $L^{2}(\mu)$ and $N$ is a unitary operator acting on $L^{2}(\mu)$ such that for each $f \in L^{1}(\mu) \cap L^{2}(\mu)$, the inequalities $\left\|N^{*} e^{i A t} N f\right\|_{1} \leqq e^{K t}\|f\|_{1},\left\|N\left(e^{i A t}\right) * N^{*} f\right\|_{1} \leqq$ $e^{K t}\|f\|_{1}$ hold for all $t \geqq 0$. Suppose also that $N$ and $Q$ commute.

Then there exists a unique $\sigma$-additive operator valued measure $\tilde{M}_{A, t}: \sigma\left(\mathcal{S}_{t}\right) \rightarrow$ $\mathcal{L}\left(L^{2}(\mu)\right)$ defined on the $\sigma$-algebra $\sigma\left(\mathcal{S}_{t}\right)$ generated by $\mathcal{S}_{t}$ such that for each $E \in$ $\mathcal{S}_{t}$, the equality $\tilde{M}_{A, t}(E) f=M_{A, t}(E) f$ holds for all $f \in L^{2}(\mu)$. Moreover, $\left\|\tilde{M}_{A, t}(E)\right\|_{\perp(L 2(\mu))} \leqq e^{K t}$ for all $E \in \sigma\left(\mathcal{S}_{t}\right)$.

Proof. According to Proposition 1.3, for each $1 \leqq p \leqq \infty$ and $t>0$, the inequality $\left\|N^{*} M_{A, t}(E) N f\right\|_{p} \leqq e^{K t}\|f\|_{p}$ holds for all $f \in L^{p}(\mu) \cap L^{2}(\mu)$ and all sets $E$ belonging to the algebra $\alpha\left(\mathcal{S}_{t}\right)$ of sets generated by $\mathcal{S}_{t}$. Because $N$ is a unitary operator acting on $L^{2}(\mu)$, the density of $L^{p}(\mu) \cap L^{2}(\mu)$ in $L^{2}(\mu)$ ensures that $\left\|M_{A, t}(E)\right\|_{\mathcal{L}(L 2(\mu))} \leqq e^{K t}$ for all $E \in \alpha\left(\mathcal{S}_{t}\right)$, that is, $M_{A, t}$ is a bounded additive set function taking values in the space $\mathcal{L}\left(L^{2}(\mu)\right)$ of bounded linear operators on $L^{2}(\mu)$.

The $\sigma$-additivity on the algebra $\alpha\left(S_{t}\right)$ of subsets of $\Omega$ follows from [J3, Proposition 2], a result of Kolmogorov concerning projective limits of measures on $\Sigma^{I}$ [Ne, III. 3] and the Orlicz-Pettis theorem [D-U, I.4.4].

The problem reduces to showing that there exists a $\sigma$-additive extension of an operator valued measure from an algebra of sets $\alpha\left(\mathcal{S}_{t}\right)$ to the $\sigma$-algebra $\sigma\left(\mathcal{S}_{t}\right)$ it generates. Any bounded subset of $\mathcal{L}\left(L^{2}(\mu)\right)$ is relatively weakly compact because $L^{2}(\mu)$ is reflexive. An appeal to the Carathéodory-Hahn-Kluvánek extension theorem completes the proof [D-U, I.5.2].

Remarks. (i) The results of this section can be reformulated in terms of the spaces $L^{p}(\mu, H), 1 \leqq p \leqq \infty$ where $H$ is a Hilbert space.

(ii) If $e^{i A t}, t \geqq 0$ is merely a bounded group of operators on $L^{1}(\mu)$, then the space $L^{1}(\mu)$ can always be renormed so that it becomes a group of isometries. However, the pleasant properties of the spectral measure $Q$ may be lost for this new norm. In Proposition 1.3, the renorming operator commutes with $Q$.

\section{$\S 2$. Approximation of Semigroups Associated with the Dirac Operator}

The free Dirac operator may be represented in $L^{2}\left(\mathbb{R}^{3} ; \mathbb{C}^{4}\right)$ by means of the differential expression 


$$
D=c \sum_{j=1}^{3} \alpha_{\jmath} p_{\jmath}+\alpha_{4} m c^{2},
$$

where $c>0$ is the velocity of light, $m>0$ is the mass of the particle, $p_{j}=$ $\hbar / i \partial / \partial x_{j}$, and

$$
\alpha_{\jmath}=\left(\begin{array}{cc}
0 & \sigma_{\jmath} \\
\sigma_{\jmath} & 0
\end{array}\right) \quad \text { for } j=1,2,3, \quad \alpha_{4}=\left(\begin{array}{rr}
\sigma_{0} & 0 \\
0 & -\sigma_{0}
\end{array}\right) .
$$

Here $\sigma_{1}, \sigma_{2}, \sigma_{2}$ are the Pauli matrices

$$
\sigma_{1}=\left(\begin{array}{ll}
0 & 1 \\
1 & 0
\end{array}\right), \quad \sigma_{2}=\left(\begin{array}{cc}
0 & -i \\
i & 0
\end{array}\right), \quad \sigma_{3}=\left(\begin{array}{rr}
1 & 0 \\
0 & -1
\end{array}\right)
$$

and $\sigma_{0}=\left(\begin{array}{ll}1 & 0 \\ 0 & 1\end{array}\right)$ is the $2 \times 2$ identity matrix. In the sequel, units are chosen in which $c$ and $\hbar$ are equal to one.

The space $L^{2}\left(\mathbb{R}^{3} ; \mathbb{C}^{4}\right)$ is a Hilbert space with the inner product $(f, g)=$ $\int_{\mathbb{R}^{3}}(f(x), g(x)) d x$ for $f, g \in L^{2}\left(\mathbb{R}^{3} ; \mathbb{C}^{4}\right)$. For any $n=1,2, \cdots$, the space $\mathbb{C}^{n}$ is assumed to be equipped with the inner product $(a, b)=\sum_{j=1}^{n} a_{j} \bar{b}_{j}$ for $a=$ $\left(a_{1}, \cdots, a_{n}\right)$ and $b=\left(b_{1}, \cdots, b_{n}\right)$ in $\mathbb{C}^{n}$. The space $\mathbb{C}^{n}$ endowed with the $p$-norm $|a|_{p}=\left(\sum_{j=1}^{n}\left|a_{j}\right|^{p}\right)^{1 / p}, a=\left(a_{1}, \cdots, a_{n}\right)$ is denoted by $\mathbb{C}_{p}^{n}$, except as just mentioned, when $p=2$ where the notation $\mathbb{C}^{n}$ is used. If $v_{n}$ is the counting measure on $\{1, \cdots, n\}$, then $\mathbb{C}_{p}^{n}=L^{p}\left(v_{n}\right)$, so the results of section one apply to the spaces $L^{p}\left(\mu ; \mathbb{C}_{p}^{n}\right)$ which may be identified with $L^{p}\left(\mu \otimes v_{n}\right)$ for all $1 \leqq p \leqq \infty$.

By virtue of the angular momentum decomposition of the Dirac operator, there exists a family $\mathscr{K}_{k, m}, k= \pm 1, \pm 2, \cdots$ and $m=-|k|,-|k|+1, \cdots,|k|-1$ of mutually orthogonal subspaces of $L^{2}\left(\mathbb{R}^{3} ; \mathbb{C}^{4}\right)$, such that $L^{2}\left(\mathbb{R}^{3} ; \mathbb{C}^{4}\right)$ is the Hilbert space direct sum of $\mathscr{H}_{k, m}$, each space $\mathscr{H}_{k, m}$ is a reducing subspace for the free Dirac operator $D$ and in each $\mathscr{H}_{k, m}, D$ is unitarily equivalent to the closure in $L^{2}\left(\mathbb{R}_{+} ; \mathbb{C}^{2}\right)$, of the operator $\tau_{k}$ defined by $(0.1)$ on the space $C_{0}^{\infty}\left(\mathbb{R}_{+} ; \mathbb{C}^{2}\right)$ of smooth $\mathbb{C}^{2}$-valued functions with compact support. The essential selfadjointness of the operator $D$ with domain $C_{c}^{\infty}\left(\mathbb{R}^{3} \backslash\{0\} ; \mathbb{C}^{4}\right)$ serves to establish the essential selfadjointness in $L^{2}\left(\mathbb{R}_{+} ; \mathbb{C}^{2}\right)$ of the operator $\tau_{k}$ for each $k= \pm 1$, $\pm 2 \cdots$. A convenient reference for these and other standard facts from the spectral theory of differential operators is [W]. The details are laid out more explicitly in [A]. Thus, for each $k= \pm 1, \pm 2, \cdots$ and $m=-|k|,-|k|+1, \cdots$, $|k|-1$, we have $\tau_{k}=\mathcal{U}_{k, m}\left(D \mid \mathscr{H}_{k, n}\right) \mathcal{U}_{k, m} *$ for a unitary operator $\mathcal{U}_{k, m}: \mathscr{H}_{k, m} \rightarrow$ $L^{2}\left(\mathbb{R}_{+} ; \mathbb{C}^{2}\right)$. Here $D \mid \mathscr{H}_{k, m}$ is the restriction of $D$ to the reducing subspace $\mathscr{K}_{k, m}$. Let $\theta=\chi_{(0, \infty)}$ on $\mathbb{R}$.

2.1 Lemma. Let $1 \leqq p<\infty$. Let $\mathscr{D}\left(T_{ \pm}\right)$be the collection of all functions $f \in$ $L^{p}\left(\mathbb{R}_{+} ; \mathbb{C}_{p}^{2}\right)$ such that $f$ is absolutely continuous on all bounded subintervals of $\mathbb{R}_{+}, f^{\prime} \in L^{p}\left(\mathbb{R}_{+} ; \mathbb{C}_{p}^{2}\right)$ and $f_{2}(0)=0$ for $T_{+}$and $f_{1}(0)=0$ for $T_{-}$. Let $T_{ \pm}: \mathscr{D}\left(T_{ \pm}\right)$ $\rightarrow L^{p}\left(\mathbb{R}_{+} ; \mathbb{C}_{p}^{2}\right)$ be the linear operator defined by $T_{ \pm} f= \pm\left(\begin{array}{rr}1 & 0 \\ 0 & -1\end{array}\right) f^{\prime}$ for all $f \in$ 
$\mathscr{D}\left(T_{ \pm}\right)$. Then $T_{+}$is the infinitesimal generator of the $\mathrm{C}_{0}$-contraction semigroup on $L^{p}\left(\mathbb{R}_{+} ; \mathbb{C}_{p}^{2}\right)$ mapping $f \in L^{p}\left(\mathbb{R}_{+} ; \mathbb{C}_{p}^{2}\right)$ to the function $x \mapsto\left(\begin{array}{c}f_{1}(x+t) \\ \left(\theta f_{2}\right)(x-t)\end{array}\right)$, for almost all $x \geqq 0$, and $T_{-}$is the infinitesimal generator of the $\mathrm{C}_{0}$-contraction semigroup on $L^{p}\left(\mathbb{R}_{+} ; \mathbb{C}_{p}^{2}\right)$ mapping $f \in L^{p}\left(\mathbb{R}_{+} ; \mathbb{C}_{p}^{2}\right)$ to the function $x \mapsto\left(\begin{array}{c}\left(\theta f_{1}\right)(x-t) \\ f_{2}(x+t)\end{array}\right)$, for almost all $x \geqq 0$.

For each $t \geqq 0, e^{T-t} e^{T_{+} t}=Q_{t}, e^{T+t} e^{T_{-} t}=P_{t}$, where $Q_{t}, P_{t}: L^{p}\left(\mathbb{R}_{+} ; \mathbb{C}_{p}^{2}\right) \rightarrow$ $L^{p}\left(\mathbb{R}_{+} ; \mathbb{C}_{p}^{2}\right)$ are the projection operators given by $\left(Q_{t} g\right)(x)=\left(\begin{array}{c}\theta(x-t) g_{1}(x) \\ g_{2}(x)\end{array}\right)$, $\left(P_{t} g\right)(x)=\left(\begin{array}{c}g_{1}(x) \\ \theta(x-t) g_{2}(x)\end{array}\right)$, for $x \geqq 0$ and $g \in L^{p}\left(\mathbb{R}_{+} ; \mathbb{C}_{p}^{2}\right)$. The operator $T_{+}$on $L^{p}\left(\mathbb{R}_{+} ; \mathbb{C}_{p}^{2}\right)$ is the Banach space adjoint of $T_{-}$on $L^{q}\left(\mathbb{R}_{+} ; \mathbb{C}_{q}^{2}\right), 1 / p+1 / q=1$. Moreover, $T_{+}$is the Hilbert space adjoint of $T_{-}$.

Proof. Let $1 \leqq p<\infty$ and for each $f \in L^{p}\left(\mathbb{R}_{+} ; \mathbb{C}_{p}^{2}\right)$, set $(S(t) f)(x)=$ $\left(\begin{array}{c}f_{1}(x+t) \\ \left(\theta f_{2}\right)(x-t)\end{array}\right)$ for almost all $x \geqq 0$. Then for each $t \geqq 0,\|S(t) f\|_{p}=\left[\int_{0}^{\infty}\left|f_{1}(x+t)\right|^{p}\right.$ $\left.+\left|\theta(x-t) f_{2}(x-t)\right|^{p} d x\right]^{1 / p} \leqq\|f\|_{p}$, in view of the assumption that $\mathbb{C}_{p}^{2}$ is equipped with the $l^{p}$-norm. Because $(S(t) f)_{2}(0)=0$ for all $f \in L^{p}\left(\mathbb{R}_{+} ; \mathbb{C}_{p}^{2}\right)$ and $t>0$, if follows that $f_{2}(0)=0$ for all functions $f$ belonging to the domain $\mathscr{D}\left(T_{+}\right)$of the infinitesimal generator $T_{+}$of $S$. The identification of $T_{+}$follows from Lebesgue's differentiation theorem. The identification of $T$ - follows in a similar manner.

The remainder of the statement involves straightforward calculations.

Remark. Neither $e^{T_{-} t}$ nor $e^{T_{-} t}$ is a contraction semigroup on $L^{p}\left(\mathbb{R}_{+} ; \mathbb{C}^{2}\right)$

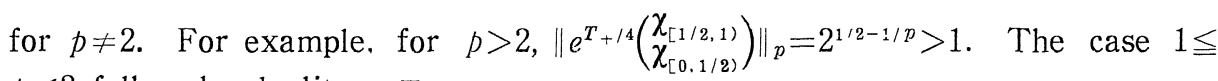
$p<2$ follow by duality.

Let $N=1 / \vee^{\prime} \overline{2}\left(\begin{array}{ll}1 & i \\ i & 1\end{array}\right)$. Then $\imath N^{*}\left(\begin{array}{cc}0 & -1 \\ 1 & 0\end{array}\right) N=\left(\begin{array}{cc}1 & 0 \\ 0 & -1\end{array}\right)$. Let $\mathscr{D}\left(\tau_{+}\right)$be the collection of all functions $f \in L^{2}\left(\mathbb{R}_{+} ; \mathbb{C}^{2}\right)$ such that $f$ is absolutely continuous on all bounded subintervals of $\mathbb{R}_{+}, f^{\prime} \in L^{2}\left(\mathbb{R}_{+} ; \mathbb{C}^{2}\right)$ and $\left(N^{*} f\right)_{2}(0)=0$. Let $\mathscr{D}\left(\tau_{-}\right)$ be the collection of all functions $f \in L^{2}\left(\mathbb{R}_{+} ; \mathbb{C}^{2}\right)$ such that $f$ is absolutely continuous on compact subsets of $\mathbb{R}_{+}, f^{\prime} \in L^{2}\left(\mathbb{R}_{+} ; \mathbb{C}^{2}\right)$ and $\left(N^{*} f\right)_{1}(0)=0$. Let $\tau_{ \pm}$: $\mathscr{D}\left(\tau_{ \pm}\right) \rightarrow L^{2}\left(\mathbb{R}_{+} ; \mathbb{C}^{2}\right)$ be the operator $\pm\left(\begin{array}{cc}0 & -1 \\ 1 & 0\end{array}\right) d / d x$.

2.2 Lemma. The operators $i \tau_{ \pm}$are the generators of $\mathrm{C}_{0}$-semigroups on $L^{p}\left(\mathbb{R}_{+} ; \mathbb{C}_{p}^{2}\right)$ given by $e^{\imath \tau}{ }_{-}^{t}=N e^{T_{\perp} t} N^{*}$ such that $\left\|e^{i \tau_{ \pm} t}\right\|_{\mathcal{L}\left(L^{p}\left(\mathbb{R}_{+} ; \mathbb{C}_{p}^{2}\right)\right)} \leqq 2$ for all $t \geqq 0$.

Proof. The resolvents of the semigroups $t \mapsto e^{i \tau} \pm^{t}, t \geqq 0$ and $t \mapsto N e^{T_{ \pm} t} N^{*}$, $t \geqq 0$ are equal, so the equality of the two semigroups follows from the uniqueness theorem for Laplace transforms [H-P, Theorem 6.2.3]. By Lemma 2.1, the norm inequality $\left\|e^{i \tau_{\Perp} t}\right\|_{\mathcal{L}\left(L^{p}\left(\mathbb{R}_{+} ; \mathrm{C}_{p}^{2}\right)\right)} \leqq\|N\|_{\mathcal{L}\left(\mathrm{C}_{p}^{2}\right)}\left\|N^{*}\right\|_{\mathcal{L}\left(\mathrm{C}_{p}^{2}\right)} \leqq 2$ holds. 
2.3 Proposition. Let $q: \mathbb{R}_{+} \rightarrow \mathbb{R}$ be locally square integrable on $(0, \infty)$. Let $\mathscr{D}\left(H_{+}\right)$be the collection of all functions $f \in L^{2}\left(\mathbb{R}_{+} ; \mathbb{C}^{2}\right)$ absolutely continuons on all bounded subintervals of $\mathbb{R}_{+}$, such that $\left(N^{*} f\right)_{2}(0)=0$ and $H_{+} f \equiv \tau_{+} f-q f \in$ $L^{2}\left(\mathbb{R}_{+} ; \mathbb{C}^{2}\right)$. Similarly, let $\mathscr{D}\left(H_{-}\right)$be the collection of all functions $f \in L^{2}\left(\mathbb{R}_{+} ; \mathbb{C}^{2}\right)$ absolutely continuous on all bounded subintervals of $\mathbb{R}_{+}$, such that $(N * f)_{1}(0)=0$ and $H_{-} f \equiv \tau_{-} f+q f \in L^{2}\left(\mathbb{R}_{+} ; \mathbb{C}^{2}\right)$.

Then $i H_{+}$is the infinitesimal generator of the $\mathrm{C}_{0}$-contraction semigroup $e^{i H_{+} t}$ on $L^{2}\left(\mathbb{R}_{+} ; \mathbb{C}^{2}\right)$, such that $e^{i H_{+} t}$ maps $f \in L^{2}\left(\mathbb{R}_{\tau} ; \mathbb{C}^{2}\right)$ to the function

$$
x \mapsto N\left(\begin{array}{c}
\exp \left[-i \int_{0}^{t} q(x+s) d s\right]\left(N^{*} f\right)_{1}(x+t) \\
\theta(x-t) \exp \left[-i \int_{0}^{t} \theta(x-t) q(x-s) d s\right]\left(N^{*} f\right)_{2}(x-t)
\end{array}\right) \text {, for almost all } x>0 .
$$

Similarly, $i H_{-}$is the infinitesimal generator of the $\mathrm{C}_{0}$-contraction semigroup $e^{i H-t}$, such that $e^{i H_{-} t}$ maps $f \in L^{2}\left(\mathbb{R}_{+} ; \mathbb{C}^{2}\right)$ to the function

$$
x \mapsto N\left(\begin{array}{c}
\theta(x-t) \exp \left[i \int_{0}^{t} \theta(x-t) q(x-s) d s\right]\left(N^{*} f\right)_{1}(x-t) \\
\exp \left[i \int_{0}^{t} q(x+s) d s\right]\left(N^{*} f\right)_{2}(x+t)
\end{array}\right) \text {, for almost all } x>0 .
$$

Proof. The statement is verified for $H_{+}$. The rest of the conclusion for $H_{-}$follows in the analogous manner.

Dominated convergence and the local integrability of $q$ ensures that the expression defining $e^{i H_{+} t}$ gives a $\mathrm{C}_{0}$-semigroup of operators on $L^{2}\left(\mathbb{R}_{+} ; \mathbb{C}^{2}\right)$. Let $\mathscr{D}$ be the range of the resolvent operator $R(\lambda)=\int_{0}^{\infty} e^{-\lambda t} e^{i H_{+} t} d t$ of the $\mathrm{C}_{0}$-semigroup $e^{i H_{+} t}, t \geqq 0$. The range $\mathscr{D}$ is independent of $\lambda \in \mathbb{C}, \operatorname{Re}(\lambda)>0$. Let $f \in L^{2}\left(\mathbb{R}_{+} ; \mathbb{C}^{2}\right)$ and set $g=N^{*} f$. Then for $x>a>0$,

$$
\begin{aligned}
\left(N^{*} R(\lambda) f\right)_{1}(x) & =\int_{0}^{\infty} e^{-\lambda t} \exp \left[-i \int_{0}^{t} q(x+s) d s\right] g_{1}(x+i) d t \\
& =\exp \left[i \int_{a}^{x} q(s) d s\right] e^{\lambda x} \int_{x}^{\infty} e^{-\lambda u} \exp \left[-i \int_{a}^{u} q(s) d s\right] g_{1}(u) d u .
\end{aligned}
$$

The function $\left(N^{*} R(\lambda) f\right)_{1} \in L^{2}\left(\mathbb{R}_{+} ; \mathbb{C}^{2}\right)$ is plainly absolutely continuous on bounded subintervals of $\mathbb{R}_{+}$. A similar argument applies to $(N * R(\lambda) f)_{2}$. Because $\left(N^{*} e^{i H_{+} t} f\right)_{2}(0)=0$ for all $t>0$, we have $\left(N^{*} R(\lambda) f\right)_{2}(0)=0$ as well. A straightforward calculation shows that $H_{+} R(\lambda) f \equiv \tau_{+} R(\lambda) f-q R(\lambda) f \in L^{2}\left(\mathbb{R}_{+} ; \mathbb{C}^{2}\right)$, so $\mathscr{D} \subseteq$ $\mathscr{D}\left(H_{+}\right)$and the restriction of $H_{+}$to $\mathscr{D}$ is the infinitesimal generator of $e^{i H_{+} t}$. Integration by parts shows that $i R(\lambda) H_{+} f=\lambda R(\lambda) f-f$ for all $f \in \mathscr{D}\left(H_{+}\right)$, so $\mathscr{D}\left(H_{+}\right) \subseteq \mathscr{D}$ and $H_{+}$is actually the infinitesimal generator of $e^{i H_{+} t}$.

For every $\varepsilon \geqq 0$, let $U_{k, \varepsilon}(r)=\left(\begin{array}{cc}m & -k r^{-1} \\ -k r^{-1} & -m\end{array}\right)$ for $r \geqq \varepsilon$ and $U_{k, \varepsilon}(r)=$ $\left(\begin{array}{cc}m & -k \varepsilon^{-1} \\ -k \varepsilon^{-1} & -m\end{array}\right)$ for $0<r<\varepsilon$. Then $U_{k, \varepsilon}$ is a bounded function with values in 
the $2 \times 2$ hermitian matrices. According to [K, Theorem IX.2.1], $i\left(\tau_{+}+U_{k, \varepsilon}\right)$ and $i\left(\tau_{-}-U_{k, \varepsilon}\right)$ are the generators of $\mathrm{C}_{0}$-semigroups on $L^{p}\left(\mathbb{R}_{+} ; \mathbb{C}_{p}^{2}\right)$ for each $\varepsilon>0$ and $1 \leqq p<\infty$. In the case $p=2$, the operators $\imath\left(\tau_{+}+U_{k, \varepsilon}\right)$ and $i\left(\tau_{-}-U_{k, \varepsilon}\right)$ are the generators of $\mathrm{C}_{0}$-contraction semigroups on $L^{2}\left(\mathbb{R}_{+} ; \mathbb{C}^{2}\right)$ for each $\varepsilon>0$.

The following results are stated for the operator $\tau_{+}$, but the analogous statements also hold for $\tau_{-}$. Let $\tau_{k, \varepsilon}=\tau_{+}+U_{k, \varepsilon}$. The same symbol $e^{i \tau_{k, \varepsilon} t}, t \geqq 0$ is used on each space $L^{p}\left(\mathbb{R}_{+} ; \mathbb{C}_{p}^{2}\right)$. By virtue of $[\mathrm{K}$, Theorem IX.2.1], the mapping $\varepsilon \mapsto e^{i=k, \varepsilon^{t}}, \varepsilon>0$ is continuous on the open interval $(0, \infty)$, in the strong operator topology of $\mathcal{L}\left(L^{2}\left(\mathbb{R}_{+} ; \mathbb{C}^{2}\right)\right)$, uniformly for $t$ in compact subsets of $[0, \infty)$.

The operator $\tau_{k}$ defined by $(0.1)$ is essentially selfadjoint; its closure in $L^{2}\left(\mathbb{R}_{+} ; \mathbb{C}^{2}\right)$ is denoted by the same symbol. A result of Trotter [T, Theorem 5.2] ensures that $\lim _{\varepsilon \rightarrow 0} e^{i \tau_{k, \varepsilon} t}=e^{i \tau_{k} t}$ in the strong operator topology of $\left.\mathcal{L}\left(L^{2} \mathbb{R}_{+} ; \mathbb{C}^{2}\right)\right)$, uniformly for $t$ in compact subsets of $[0, \infty)$. The operator $\tau_{k, 0}$ is set equal to $\tau_{k}$.

2.4 Lemma. The norm $\left\|N^{*} e^{i \tau_{k, \varepsilon^{l}}} N\right\|$ of the operator $N^{*} e^{i \tau_{k, \varepsilon^{l}} N}$ on $L^{1}\left(\mathbb{R}_{+}\right.$; $\left.\mathbb{C}_{1}^{2}\right)$ is bounded by $e^{(n+|k| / s) t}$. Similarly, $\| N\left(e^{2 \tau k, \varepsilon t) * N^{*} \|_{L}\left(L_{1}^{1}\left(\mathbb{R}_{+} ; \mathbb{C}_{1}^{2}\right)\right) \leqq} e^{(m+|k| / s) t}\right.$.

Proof. A calculation shows that $N * U_{k, \varepsilon}(r) N=\left(\begin{array}{cc}0 & i m-k / r \\ -i m-k / r & 0\end{array}\right)$ for $r>\varepsilon$ and $N^{*} U_{k, \varepsilon}(r) N=\left(\begin{array}{cc}0 & i m-k / \varepsilon \\ -i m-k / \varepsilon & 0\end{array}\right)$ for $r \leqq \varepsilon$, so $\left\|N^{*} U_{k, \varepsilon}(r) N\right\|_{\mathrm{C}_{1}^{2} \leqq m}$ $+|k| / r$ for $r>\varepsilon$ and $\left\|N^{*} U_{k, \varepsilon}(r) N\right\|_{c_{1}^{2}} \leqq m+|k| / \varepsilon$ for $r \leqq \varepsilon$. Because $N^{*} \tau_{+} N=$ $T_{+}, N^{*} e^{\imath \tau} \tau_{k, \varepsilon} t N, t \geqq 0$ is the $\mathrm{C}_{0}$-semigroup generated by $T_{+}+N * U_{k, \varepsilon}(r) N$. As noted in Lemma $2.1, T_{+}$is the generator of a contraction semigroup on $L^{1}\left(\mathbb{R}_{+} ; \mathbb{C}_{1}^{2}\right)$, so the result follows from [K, Theorem IX.2.1]. The estimate for the adjoint operator is proved similarly.

2.5 Lemma. Suppose that $q: \mathbb{R}_{+} \rightarrow \mathbb{R}$ is locally integrable on $[0, \infty)$ and locally square integrable on $(0, \infty)$. Let $q_{n}=q \chi_{i q 1 \leqq n}, n=1,2, \cdots$. Then for every $\lambda>0$, the function $s \mapsto\left(\lambda-i\left(\tau_{k, \varepsilon}-q_{n}\right)\right)^{-1}, 0 \leqq \varepsilon \leqq 1$ is continuous in the strong operator topology of $\mathcal{L}\left(L^{2}\left(\mathbb{R}_{+} ; \mathbb{C}^{2}\right)\right)$, uniformly for $n=1,2, \cdots$. Furthermore, as $\varepsilon \rightarrow 0^{+}$, $e^{i\left(\tau_{\left.k, \varepsilon^{-} q_{n}\right) t} \rightarrow e^{\imath\left(\tau_{k}-q_{n}\right) t}\right.}$ in the strong operator topology of $\mathcal{L}\left(L^{2}\left(\mathbb{R}_{+} ; \mathbb{C}^{2}\right)\right)$, uniformly for $n=1,2, \cdots$ and $t$ in compact subsets of $[0, \infty)$.

Proof. Let $X$ be the Banach space of all continuous functions $f:[0,1] \rightarrow$ $L^{2}\left(\mathbb{R}_{+} ; \mathbb{C}^{2}\right)$ with the norm $\|f\|_{\infty}=\sup _{0 \leqq x \leqq 1}\|f(x)\|_{2}$. For each $t \geqq 0$ and $f \in X$, set $(S(t) f)(\varepsilon)=e^{i \tau_{k, \varepsilon} l} f(\varepsilon)$ for all $0 \leqq \varepsilon \leqq 1$. Then $S(t) f \in X$ and $S(s) S(t)=S(t+s)$ for all $s \geqq 0$. Moreover, as noted above, $\varepsilon \mapsto e^{\imath \tau} \varepsilon_{,} t, 0 \leqq \varepsilon \leqq 1$ is continuous in the strong operator topology, uniformly for $t$ in compact subsets of $[0, \infty)$. It follows that $t \mapsto S(t), t \geqq 0$ is a $\mathrm{C}_{0}$-semigroup acting on $X$.

Now $U_{k, \varepsilon}$ is a bounded operator on $L^{2}\left(\mathbb{R}_{+} ; \mathbb{C}^{2}\right)$, so by Proposition 2.3 and [K, Theorem IX.2.1], the operator $i\left(\tau_{k, \varepsilon}-q\right)$ is the generator of a $\mathrm{C}_{0}$-semigroup on $L^{2}\left(\mathbb{R}_{+} ; \mathbb{C}^{2}\right)$, and as before, the mapping $\varepsilon \mapsto e^{i\left(\tau_{k, \varepsilon}-q\right) t}, \varepsilon>0$ is continuous on the open interval $(0, \infty)$, in the strong operator topology of $\mathcal{L}\left(L^{2}\left(\mathbb{R}_{+} ; \mathbb{C}^{2}\right)\right.$ ), 
uniformly for $t$ in compact subsets of $[0, \infty)$.

The function $q$ is assumed to be locally integrable, so according to $[\mathrm{Be}$, Lemma 3], $\tau_{k}-q$ is selfadjoint. Another appeal to Trotter's convergence theorem [T, Theorem 5.2] ensures that $\lim _{\varepsilon \rightarrow 0} e^{2\left(\tau_{k, \varepsilon^{-}}-q\right) t}=e^{i\left(\tau_{k}-q\right) t}$ in the strong operator topology of $\mathcal{L}\left(L^{2}\left(\mathbb{R}_{+} ; \mathbb{C}^{2}\right)\right)$, uniformly for $t$ in compact subsets of $[0$, $\infty)$. For each $t \geqq 0$ and $f \in X$, set $\left(S_{q}(t) f\right)(\varepsilon)=e^{i\left(\tau_{k, \varepsilon}-q\right) t} f(\varepsilon)$ for all $0 \leqq \varepsilon \leqq 1$. The same argument as before shows that $t \mapsto S_{q}(t), t \geqq 0$ is a $\mathrm{C}_{0}$-semigroup acting on $X$.

The set $C_{c}^{\infty}\left((0, \infty), \mathbb{C}^{2}\right)$ is a core for $\tau_{k}-q[\mathrm{Be}$, Lemma 3$]$ and, as is easily verified, the set $C([0,1]) \otimes C_{c}^{\infty}\left((0, \infty), \mathbb{C}^{2}\right)$ of all finite linear combinations $f(\varepsilon)(r)=\sum_{j=1}^{n} g_{j}(\varepsilon) h_{j}(r), 0 \leqq \varepsilon \leqq 1, r \geqq 0$ of functions $g_{j} \in C([0,1])$ and $h_{j} \in$ $C_{c}^{\infty}\left((0, \infty), \mathbb{C}^{2}\right), j=1, \cdots, n, n=1,2, \cdots$ is a core for the infinitesimal generator of $S_{q}$. Moreover, $\left(\tau_{k, \varepsilon}-q_{n}\right) f(\varepsilon) \rightarrow\left(\tau_{k, \varepsilon}-q\right) f(\varepsilon)$ uniformly for $0 \leqq \varepsilon \leqq 1$ for any such $f \in C([0,1]) \otimes C_{c}^{\infty}\left((0, \infty), \mathbb{C}^{2}\right)$. Again, [T, Theorem 5.2] implies that as $n \rightarrow \infty, S_{q_{n}}(t) \rightarrow S_{q}(t)$ in the strong operator topology of $\mathcal{L}(X)$, uniformly for $t$ in compact subsets of $\mathbb{R}_{+}$.

For each $h \in L^{2}\left(\mathbb{R}_{+} ; \mathbb{C}^{2}\right)$ and $n=1,2, \cdots$, the function $\int_{0}^{\infty} e^{-\lambda t} S_{q_{n}}(t) \mathbf{1} h d t \in X$ is just the mapping $\varepsilon \mapsto\left(\lambda-i\left(\tau_{k, \varepsilon}-q_{n}\right)\right)^{-1} h$, where the function identically equal to one on $[0,1]$ has been denoted by 1 . As $n \rightarrow \infty$, these functions converge uniformly on $[0,1]$ to the function $\varepsilon \mapsto\left(\lambda-i\left(\tau_{k, \varepsilon}-q\right)\right)^{-1} h, 0 \leqq \varepsilon \leqq 1$. This implies the first conclusion. The second follows from the existence of the limit $\lim _{n \rightarrow \infty} S_{q_{n}}(t) \mathbf{1} h=S_{q}(t) \mathbf{1} h$ in $X$.

2.6 Corollary. Let $q_{n}, n=1,2, \cdots$ be as in Lemma 2.5. For any bounded linear operators $T_{1}, \cdots, T_{k}$ on $L^{2}\left(\mathbb{R}_{+} ; \mathbb{C}^{2}\right)$ and any numbers $s_{1}, \cdots, s_{k}>0$, $\lim _{n \rightarrow \infty} \prod_{j=1}^{k}\left[e^{i\left(\tau_{k, \varepsilon}-q_{n}\right) s} T_{j}\right]=\prod_{j=1}^{k}\left[e^{i\left(\tau_{k, \varepsilon}-q\right) s_{j}} T_{j}\right]$ in the strong operator topology of $\mathcal{L}\left(L^{2}\left(\mathbb{R}_{+} ; \mathbb{C}^{2}\right)\right)$, uniformly for $1 \leqq \varepsilon \leqq 1$.

Proof. This follows from the Banach-Steinhaus theorem, and the observation that $\bigcup_{0 \leqq \varepsilon \leqq 1, n} e^{i\left(\tau_{k, \varepsilon}-q_{n}\right) s_{j}} K, 0 \leqq \varepsilon \leqq 1$ is precompact for any precompact subset $K$ of $L^{2}\left(\mathbb{R}_{+} ; \mathbb{C}^{2}\right)$.

2.7 Lemma. Let $q: \mathbb{R}_{+} \rightarrow \mathbb{R}$ be a bounded Borel measurable function. Then as $n \rightarrow \infty$, the operators $\left[e^{-i q t / n} e^{i \tau_{k, \varepsilon} t / n}\right]^{n}, n=1,2, \cdots$ converge to $e^{i\left(\tau_{k, \varepsilon}-q\right) t}$ in the strong operator topology of $\mathcal{L}\left(L^{2}\left(\mathbb{R}_{+} ; \mathbb{C}^{2}\right)\right)$, uniformly for $0<\varepsilon \leqq a$, for each $a>0$.

Proof. The statement is a version of Trotter's product formula with the convergence uniform as the parameter $\varepsilon>0$ varies. The operators $\tau_{k, \varepsilon}, \varepsilon>0$ have a common domain $\mathscr{D}\left(\tau_{+}\right)$and the function $\varepsilon \mapsto \tau_{k, \varepsilon} x, \varepsilon>0$ is continuous for each $x \in \mathscr{D}\left(\tau_{+}\right)$, so the conclusion follows from [J2, Theorem 1$]$.

Let $C_{0}\left(\mathbb{R}_{+}, \mathbb{C}^{2}\right)$ be the space of all continuous functions $f: \mathbb{R}_{+} \rightarrow \mathbb{C}^{2}$ such that $f(x) \rightarrow 0$ as $x \rightarrow \infty$. The bilinear integrals referred to in the next statement 
are to be understood in the sense of Bartle [B], as mentioned earlier, or more simply, componentwise. The equivalence class of a Borel measurable function $g: \mathbb{R}_{+} \rightarrow \mathbb{C}^{2}$ with respect to Lebesgue measure is denoted by $[g]$.

2.8 Proposition. There exists a unique semigroup $T_{k, \varepsilon}$ on $C_{0}\left(\mathbb{R}_{+}, \mathbb{C}^{2}\right)$ such that $\left[T_{k, \varepsilon}(t) f\right]=e^{i=k, s^{t}}[f]$ for all $f \in C_{0}\left(\mathbb{R}_{+}, \mathbb{C}^{2}\right)$ and $t \geqq 0$. Furthermore, there exists a unique $2 \times 2$-matrix valued function $(t, x, B) \mapsto p_{t}(x, B), t \geqq 0, x>0, B \in$ $\mathscr{B}\left(\mathbb{R}_{+}\right)$with the following properties:

(i) for each $t \geqq 0$ and $x>0, B \mapsto p_{t}(x, B), B \in \mathscr{B}\left(\mathbb{R}_{+}\right)$is a $2 \times 2$-matrix valued measure,

(ii) for each $B \in \mathscr{B}\left(\mathbb{R}_{+}\right)$, the function $(t, x) \mapsto p_{l}(x, B), t \geqq 0, x>0$ is jointly Borel measurable, and

(iii) for each $t, s \geqq 0, x>0$ and $B \in \mathscr{B}\left(\mathbb{R}_{+}\right), p_{t+s}(x, B)=\int_{0}^{\infty} p_{s}(x, d y) p_{t}(y, B)$,

(iv) for each $t \geqq 0, x>0$ and $f \in C_{0}\left(\mathbb{R}_{+}, \mathbb{C}^{2}\right),\left(T_{k, \varepsilon} f\right)(x)=\int_{0}^{\infty} p_{l}(x, d y) f(y)$.

Proof. The dual estimate from Lemma 2.4 shows that the semigroup $e^{2 \tau} k, \varepsilon^{l}$ on $L^{\infty}\left(\mathbb{R}_{+} ; \mathbb{C}_{\infty}^{2}\right)$ satisfies $\left\|N^{*} e^{i \tau_{k, \varepsilon} t} N f\right\|_{\infty} \leqq e^{(m+|k| / s) t}\|f\|_{\infty}$ for all $f \in L^{\infty}\left(\mathbb{R}_{+} ; \mathbb{C}_{\infty}^{2}\right)$ and $t \geqq 0$. If $f \in C_{c}^{\infty}\left(\mathbb{R}_{+}, \mathbb{C}^{2}\right)$, then it is easily verified that $e^{i \tau_{k, \varepsilon} t} f \in C_{0}\left(\mathbb{R}_{+}, \mathbb{C}^{2}\right)$ (in the sense that there exists a unique continuous representative of $e^{i \tau_{k, s} t} f$ ), so continuity ensures the existence of the stated linear operators $T_{k, \varepsilon}(t): C_{0}\left(\mathbb{R}_{+}, \mathbb{C}^{2}\right)$ $\rightarrow C_{0}\left(\mathbb{R}_{+}, \mathbb{C}^{2}\right)$ for each $t \geqq 0$ and the semigroup property for $T_{k \varepsilon}$ follows from the semigroup property of $e^{i \tau_{k, \varepsilon} t}, t \geqq 0$ on $L^{\infty}\left(\mathbb{R}_{+} ; \mathbb{C}_{\infty}^{2}\right)$.

The existence and properties of the matrix valued "transition function" $(t, x, B) \rightarrow p_{t}(x, B), t \in \mathbb{R}, x>0, B \in \mathscr{B}\left(\mathbb{R}_{+}\right)$are proved in the manner analogous to that for Feller semigroups, only now we deal with matrix valued measures rather than probability measures. Put simply, the Riesz representation theorem ensures that the Banach space dual operator $T_{k, \varepsilon}(t)^{\prime}$ of $T_{k, \varepsilon}(t)$ maps the space of $\mathbb{C}^{2}$-valued Borel measures on $\mathbb{R}_{+}$into itself. Then for each $t \in \mathbb{R}, x>0$ and $B \in \mathscr{B}\left(\mathbb{R}_{+}\right)$, set $p_{t}(x, B) v=\left[T_{k}(t)^{\prime} \delta_{x} v\right](B)$ for each vector $v \in \mathbb{C}^{2}$. Here $\delta_{x}$ is the unit point mass at $x \in \mathbb{R}_{+}$. Then $(t, x, B) \mapsto p_{t}(x, B), t \in \mathbb{R}, x>0, B \in \mathscr{B}\left(\mathbb{R}_{+}\right)$ has the stated properties.

\section{§3. Existence of the Spherically Symmetric Dirac Cut-off Measures}

Denote the semi-algebra of all sets $E$ of the form (1.1) with $\Sigma=\mathbb{R}_{+}$by $R_{\iota}$ and set $\Gamma_{\infty}=\sum^{[0, \infty)}$. For each $t, \varepsilon>0$, the associated $\left(e^{\imath \tau / \varepsilon \varepsilon}, Q, t\right)$-set function is denoted by $R_{t}^{(k, s)}: \alpha\left(\mathscr{R}_{t}\right) \rightarrow \mathcal{L}\left(L^{2}\left(\mathbb{R}_{+} ; \mathbb{C}^{2}\right)\right)$. Here $Q$ is the spectral measure of multiplication by bounded Borel subsets of $\mathbb{R}_{+}$, acting on the Hilbert space $L^{2}\left(\mathbb{R}_{+} ; \mathbb{C}^{2}\right)$.

According to Lemma 2.4 and Proposition 1.3 we have the following result.

3.1 Lemma. $\sup _{A \in a\left(R_{t}\right)}\left\|R_{t}^{(k, \varepsilon)}(A)\right\|_{\mathcal{L}\left(L^{2}\left(R_{+} ; \mathrm{C} 2\right)\right)} \leqq e^{(|n+1 k| / \varepsilon) t}$. 
Moreover, it follows from Theorem 1.4 that $R_{t}^{(k, s)}$ has a unique $\sigma$-additive extension to the $\sigma$-algebra $\sigma\left(\mathscr{R}_{t}\right)$ generated by $\mathcal{R}_{t}$.

For each $k \in \mathbb{Z} \backslash\{0\}$, let $T_{k}$ be the unitary group of operators defined on $L^{2}\left(\mathbb{R}_{+} ; \mathbb{C}^{2}\right)$ by $T_{k}(t)=e^{2 \tau} k^{t}$ for all $t \in \mathbb{R}$. For each $t>0$ there exists, according to formula (1.2), an operator valued set function $R_{t}^{(k)}: \mathscr{R}_{t} \rightarrow \mathcal{L}\left(L^{2}\left(\mathbb{R}_{+} ; \mathbb{C}^{2}\right)\right)$ defined on the semi-algebra $\mathscr{R}_{t}$ of all sets $E$ of the form (1.1) with $\Sigma=\mathbb{R}_{+}$and $\left.\Gamma_{\infty}=\Sigma^{0}{ }^{\infty}\right)$, such that the restriction of $R_{t}^{(k)}$ to $\mathcal{R}_{t}$ is the $\left(T_{k}, Q, t\right)$-set function. Here $Q$ is the spectral measure acting on $L^{2}\left(\mathbb{R}_{+} ; \mathbb{C}^{2}\right)$ of multiplication by Borel subsets of $\mathbb{R}_{+}$. It will prove useful to formulate this observation in terms of an $\mathbb{R}^{3}$-valued process whose distributions are operator valued set functions acting on $L^{2}\left(\mathbb{R}^{3} ; \mathbb{C}^{4}\right)$.

Now let $\Omega_{\infty}$ be the space of all paths $\omega:[0, \infty) \rightarrow \mathbb{R}^{3}$. Let $\mathscr{B}_{\mathrm{R}}$ be the sub$\sigma$-algebra of the Borel sets in $\mathbb{R}^{3}$ consisting of radially symmetric Borel sets, that is, $\mathscr{B}_{\mathbb{R}}$ is the collection of all sets $\left\{x \in \mathbb{R}^{3}:|x| \in B\right\}, B \in \mathcal{B}\left(\mathbb{R}_{+}\right)$.

The spectral measure acting on $L^{2}\left(\mathbb{R}^{3} ; \mathbb{C}^{4}\right)$ of multiplication by characteristic functions of sets belonging to $\mathscr{B}_{\mathbf{R}}$ is denoted by $Q_{R}$. For each $t>0$, the semi-algebra of all sets of the form (1.1) with the sets $B_{1}, \cdots, B_{n}$ belonging to $\mathscr{B}_{\mathrm{R}}$ is denoted by $\mathcal{S}_{t}$. Let $S_{D_{\varepsilon}}$ be the $C_{0}$-semigroup of operators acting on $L^{2}\left(\mathbb{R}^{3} ; \mathbb{C}^{4}\right)$ and defined by $S_{D_{\varepsilon}}(t)=e^{i D_{\varepsilon} t}$ for all $t \geqq 0$, where $D_{\varepsilon}=\sum_{k, m} \oplus \mathcal{U}_{k, m} *\left(\tau_{+}\right.$ $\left.+U_{k, \varepsilon}\right) U_{k, m}$. Then $i D_{\varepsilon}$ is the generator of the $C_{0}$-contraction semigroup $S_{D_{\varepsilon}}(t)$ $=\sum_{k, m} \oplus \mathcal{U}_{k, m}{ }^{*} e^{i \tau k, \varepsilon} \mathcal{V}_{k, m}$ acting on $L^{2}\left(\mathbb{R}^{3} ; \mathbb{C}^{4}\right)$, but $D_{\varepsilon}$ is not selfadjoint. Moreover, $D_{\varepsilon} \rightarrow D$ in the strong resolvent sense.

3.2 Theorem. Let $\mathcal{S}_{t}$ be the semi-algebra of elementary events in $\Omega_{\infty}$. For each $t>0$, there exists a unique $\sigma$-additive operator valued measure $M_{t}^{(\varepsilon)}: \sigma\left(S_{t}\right)$ $\rightarrow \mathcal{L}\left(L^{2}\left(\mathbb{R}^{3} ; \mathbb{C}^{4}\right)\right)$ such that the restriction of $M_{t}^{(\varepsilon)}$ to $\mathcal{S}_{t}$ is the $\left(S_{D_{\varepsilon}}, Q_{\mathbf{R}}, t\right)$-set function.

Proof. Let $\Psi: \Omega_{\infty} \rightarrow \mathbb{R}_{+}{ }^{[0 \infty)}$ be defined by $(\Psi(\omega))(t)=|\omega(t)|$ for all $t \geqq 0$ and all $\omega \in \Omega_{\infty}$. Then for any $t>0, \mathcal{S}_{t}=\left\{\Psi^{-1}(E): E \in \mathscr{R}_{t}\right\}$.

Let $Q$ be the spectral measure of multiplication by characteristic functions of Borel sets, acting on $L^{2}\left(\mathbb{R}_{+} ; \mathbb{C}^{2}\right)$. Then for all indices $k, m$, the spectral measure $Q_{\mathrm{R}}$ satisfies

$Q_{\mathbf{R}}\left(\left\{x \in \mathbb{R}^{3}:|\ddot{x}| \in B\right\}\right) f=\mathcal{U}_{k, m} * Q(B) \mathcal{U}_{k, m} f$, for all $B \in \mathscr{B}\left(\mathbb{R}_{+}\right)$and $f \in \mathcal{H}_{k, m}$, with respect to the unitary operators $U_{k, m}$ defined earlier, so a simple calculation shows that for all $t>0$ and $E \in \mathcal{R}_{t}$, the $\left(S_{D_{\varepsilon}} Q_{\mathrm{R}}, t\right)$-set function $M_{t}^{(\varepsilon)}$ is given by $M_{t}^{(\varepsilon)}\left(\Psi^{-1}(E)\right)=\sum_{k, m} \oplus \mathcal{U}_{k, m} * R_{t}^{(k, \varepsilon)}(E) \mathcal{U}_{k, m}$. The result follows immediately.

Remarks. (i) The notation $L^{1}\left(\mathbb{R}_{+} ; \mathbb{C}_{1}^{2}\right)$ used in Lemma 2.4 is not just pendantry. It was noted earlier that neither $T_{+}$nor $T_{-}$is the generator of a contraction semigroup on $L^{1}\left(\mathbb{R}_{+}, \mathbb{C}^{2}\right)$. This difficulty actually precludes us from 
deducing the boundedness of the $\left(S_{D}, Q_{\mathrm{R}}, t\right)$-set functions as follows: if, say, $T_{+}$were perturbed by $i U$ for an hermitian matrix multiplication operator $U(r)$, and if $e^{T+t}, t \geqq 0$ were a contraction semigroup on $L^{1}\left(\mathbb{R}_{+} ; \mathbb{C}^{2}\right)$, then the inequality $\left\|\left(e^{T+t / n} e^{i U t / n}\right)^{n}\right\|_{\perp\left(L 1\left(\mathbb{R}_{+} ; C^{2}\right)\right)} \leqq 1$ would obtain for all $n=1,2, \cdots$. If, in addition, the range of the operator $\lambda-i\left(T_{+}+U\right)$ were dense in $L^{1}\left(\mathbb{R}_{+} ; \mathbb{C}^{2}\right)$, then general results on semigroup convergence $[\mathrm{T}]$ would ensure that the closure of $T_{+}+U$ generates a contraction semigroup on $L^{1}\left(\mathbb{R}_{\tau} ; \mathbb{C}^{2}\right)$. A slight variant of Proposition 1.3 would then allow us to conclude that the $\left(S_{D}, Q_{\mathrm{R}}, t\right)$-set functions acting on $L^{2}\left(\mathbb{R}^{3} ; \mathbb{C}^{4}\right)$ are actually bounded; the use of the appropriate norms on $\mathrm{C}^{n}$ is therefore crucial.

(ii) Proposition 2.8 show that for each $k \in Z \backslash\{0\}$, there exist matrix valued measures $P_{t}^{(k, s)}: \sigma\left(\mathscr{R}_{t}\right) \rightarrow \mathcal{L}\left(\mathcal{C}^{2}\right), x>0$ such that for each bounded measurable function $f: \mathbb{R}_{+} \rightarrow \mathrm{C}^{2}$ with compact support and each bounded, scalar valued, cylinder function $F$, the equality

$$
\left(R_{t}^{(k, \varepsilon)}(F) f\right)(x)=\int_{\Gamma_{\infty}} d P_{t}^{(k x} x^{s)}(\omega) f(\omega(t)) F(\omega)
$$

holds for almost all $x>0$. Here $\Gamma_{\infty}=\mathbb{R}_{+}{ }^{[0 \infty)}$. The sum of the operator valued measures $\left.\mathcal{U}_{k, m}{ }^{*} R_{t}^{(k)}{ }^{s}\right) \mathcal{U}_{k, \eta}, k \in \mathbb{Z} \backslash\{0\}$ converges in $\mathcal{L}\left(L^{2}\left(\mathbb{R}^{3} ; \mathbb{C}^{1}\right)\right)$, but there is no corresponding result for the matrix valued measures $P_{t}^{(k, x \varepsilon)}$.

\section{$\S 4$. The Support of the Spherically Symmetric Dirac Cut-off Measures}

In this section, we show that there is a distinguished subset $\Omega$ of the set $\Omega_{\infty}$ of all paths in $\mathrm{R}^{3}$ such that for any $A \in \sigma\left(\mathcal{S}_{t}\right)$ disjoint from $\Omega, M_{t}^{(\varepsilon)}(B)=0$ for all sets $B \in \sigma\left(\mathcal{S}_{t}\right)$ such that $B \subseteq A$ and all $\varepsilon>0$. It follows that for each $\varepsilon>0$, the operator valued set function $A_{1}>\Omega \rightarrow M_{t}^{(s)}(A),-1 \in \sigma\left(\mathcal{S}_{t}\right)$ is well defined and $\sigma$-additive. It is in this sense that $\Omega$ is the support of $M_{t}^{(\varepsilon)}$.

In this section the operator $\tau_{+}$defined in section two is merely denoted by $\tau$. The following argument is similar to that of [I-T2, Lemma 4.4]. By iterating the formula $e^{i\left(\tau+U_{k, \varepsilon}\right) t}=e^{\imath \tau t}+i \int_{0}^{t} e^{i \tau(l-a)} U_{k} e^{\imath\left(\tau+U_{k, \varepsilon) a}\right.} d \alpha$ it follows that the "Dyson series"

$$
e^{i\left(\tau+U_{k, \varepsilon}\right) t}=e^{\imath \tau t}+\sum_{j=1}^{\infty} i^{j} \int_{0}^{t} \cdots \int_{0}^{n_{2}} e^{i \tau\left(t-n_{\jmath}\right)} U_{k, \varepsilon} \cdots e^{i \tau\left(n_{2}-a_{1}\right)} U_{k, \varepsilon} e^{i \tau n_{1}} d \alpha_{1} \cdots d \alpha
$$

converges in the uniform operator norm (see the proof of [K, IX.2.1]).

Let $V_{0}^{(s)}(t)=e^{i \tau t}$ and $V_{j}^{(s)}(t)=\int_{0}^{t} \cdots \int_{0}^{a_{2}} e^{i \tau\left(t-\alpha_{\jmath}\right)} U_{k s} \cdots e^{i \tau\left(a_{2}-a_{1}\right)} U_{k, \varepsilon} e^{i \tau a_{1}} d \alpha_{1} \cdots d \alpha_{\jmath}$ for all $t>0$ and $j=1,2, \cdots$. Then $\left\|V_{j}^{(\varepsilon)}(t)\right\| \leqq\left\|U_{k, \varepsilon}\right\|^{j} t^{j} j !$.

The equality $e^{\imath\left(\tau+z U_{k, \varepsilon}\right)(t+s)}=e^{i\left(\tau-z U_{k, \varepsilon}\right) t} e^{i\left(\tau+z U_{k, \varepsilon}\right) s}$ is true for all $z \in \mathbb{C}$ [K, IX.2.1]. Equating the coefficients of $z^{m}, m=0,1,2 \cdots$ ensures that

$$
\sum_{l+j=m} V_{l}^{(s)}(t) V_{j}^{(\varepsilon)}(s)=V_{m}^{(\varepsilon)}(t+s)
$$


for all $s, t>0$. The sum is over integers $l, j \geqq 0$. If we set

$$
R_{t, J}^{(k, \xi)}(E)=\sum_{j_{0}+\cdots+j_{n}=J} V_{j_{n}}^{(\varepsilon)}\left(t-t_{n}\right) Q\left(B_{n}\right) V_{J_{n-1}}^{(\varepsilon)}\left(t_{n}-t_{n-1}\right) \cdots V_{j_{1}}^{(\varepsilon)}\left(t_{2}-t_{1}\right) Q\left(B_{1}\right) V_{j_{0}}^{(\varepsilon)}\left(t_{1}\right)
$$

for each set $E$ of the form (1.1) and $J=0,1,2, \cdots$, then it follows from equation (4.2) that the set function $R_{t, J}^{(k, \varepsilon)}$ is well defined and additive on the semialgebra $R_{t}$ of subsets of $\mathbb{R}_{+}{ }^{[0, \infty)}$. The sum is over integers $j_{0}, \cdots, j_{n} \geqq 0$. By (4.1), the equality

$$
R_{t}^{(k, \varepsilon)}(A)=\sum_{J=0}^{\infty} i^{J} R_{t, J}^{(k, \varepsilon)}(A)
$$

holds for all $A \in \alpha\left(\mathscr{R}_{t}\right)$. The convergence is in the uniform operator norm.

Furthermore, it follows as in Proposition 1.3 that for each $J=0,1, \cdots$, that the additive set function $R_{t, J}^{(k, \varepsilon)}$ is bounded on $\alpha\left(R_{t}\right)$ with norm $\sup _{E \in \alpha\left(\varsigma_{t}\right)}\left\|R_{t, J}^{(k, \varepsilon)}(E)\right\|_{\mathcal{L}\left(L 2\left(\mathbf{R}_{+} ; \mathrm{C}^{2}\right)\right)} \leqq a^{J} t^{J} / J$ !, where $a=\left\|N^{*} U_{k, \varepsilon} N\right\|_{\mathcal{L}\left(L_{1}\left(\mathbf{R}_{+} ; \mathrm{C}_{1}^{2}\right)\right)} \leqq$ $m+|k| / \varepsilon$.

Because each set function $R_{t, J}^{(k, \varepsilon)}, \varepsilon>0, J=0,1,2, \cdots$ is bounded on the algebra $\alpha\left(\Omega_{t}\right)$, it follows as in the proof of Theorem 1.4, that $R_{t, J}^{(k, \varepsilon)}$ is the restriction to $\alpha\left(\mathscr{R}_{t}\right)$ of a unique $\mathcal{L}\left(L^{2}\left(\mathbb{R}_{+} ; \mathbb{C}^{2}\right)\right)$-valued measure on $\sigma\left(\mathscr{R}_{t}\right)$, which is denoted by $R_{t, J}^{(k, \varepsilon)}$ as well. To examine the support properties of the $R_{t, J}^{(k, \varepsilon)}$, some additional notation is needed. In this context, it is sometimes useful to consider set functions defined on $\mathcal{R}_{t}, \alpha\left(\mathscr{R}_{t}\right), \sigma\left(\mathscr{R}_{t}\right)$ as being defined on subsets of $\mathbb{R}_{+}{ }^{[0, t]}$ instead of $\mathbb{R}_{+}{ }^{[0, \infty)}$. Because there is a one-to-one correspondence between the semi-algebras $\mathscr{R}_{t}$ and $\mathscr{R}_{t} \cap \mathbb{R}_{+}{ }^{[0, t]}$, we shall pass from set functions defined on one family of sets to the other without further mention. The same cavalier attitude is adopted for the algebras and $\sigma$-algebras they generate.

For each $J=1,2, \cdots$, let $\Delta_{J}$ be the collection of all $J$-tuples $\left(s_{1}, \cdots, s_{J}\right)$ of numbers such that $0<s_{1}<\cdots<s_{J} \leqq t$. Let $\Xi_{J}$ be the collection of all maps $\xi$ : $\{0,1, \cdots, J\} \rightarrow\{0,1\}$. Define the map $\Lambda_{J}: \mathbb{R}_{+} \times \Delta_{J} \times \Xi_{J} \rightarrow \mathbb{R}^{[0, \infty)}$ for $x>0,\left(s_{1}, \cdots\right.$, $\left.s_{J}\right) \in \Delta_{J}$ and $\xi \in \Xi_{J}$ by $\Lambda_{J}\left(\left(x,\left(s_{1}, \cdots, s_{J}\right), \xi\right)\right)=\gamma$, where $\gamma:[0, \infty) \rightarrow \mathbb{R}$ is the polygonal path defined by $\gamma(s)=x+(-1)^{\xi(0)} s$ for $0 \leqq s \leqq s_{1}$ and

$$
\gamma(s)=x+(-1)^{\xi(0)} s_{1}+\sum_{k=1}^{\jmath}(-1)^{\xi(k)}\left(s_{k+1}-s_{k}\right)+(-1)^{\xi(j+1)}\left(s-s_{\jmath+1}\right)
$$

for $s_{\jmath+1} \leqq s \leqq s_{\jmath+2}, j=0, \cdots, J-1$, with $s_{J+1}$ defined to be $t$. Let $j_{0}, \cdots, j_{n}$ be non-negative integers and $T\left(j_{0}, \cdots, j_{n} ; t_{1}, \cdots, t_{n}\right)$ the set of all $\left(s_{1}, \cdots, s_{J}\right)$ such that

$$
\begin{gathered}
0<s_{1}<\cdots<s_{\jmath_{0}} \leqq t_{1}, \\
t_{1}<s_{j_{0}+1}<\cdots<s_{\jmath_{0}+\jmath_{1}} \leqq t_{2}, \\
\vdots \\
t_{n}<s_{\jmath_{0}+\cdots+\jmath_{n-1}+1}<\cdots<s_{\jmath_{0}+\cdots+\jmath_{n}} \leqq t,
\end{gathered}
$$

with the appropriate inequality omitted if $j_{k}=0$. Writing $T=T\left(j_{0}, \cdots, j_{n}\right.$; $\left.t_{1}, \cdots, t_{n}\right)$, we have 


$$
\begin{aligned}
& V_{j_{n}}^{(\varepsilon)}\left(t-t_{n}\right) Q\left(B_{n}\right) V_{j_{n-1}}^{(\varepsilon)}\left(t_{n}-t_{n-1}\right) \cdots V_{j_{1}}^{(\varepsilon)}\left(t_{2}-t_{1}\right) Q\left(B_{1}\right) V_{j_{0}}^{(\varepsilon)}\left(t_{1}\right) \\
& =\int_{T} P_{n}\left(\alpha_{j_{0}+\cdots+\jmath_{n-1}+1}, \cdots, \alpha_{\jmath_{0}+\cdots+\jmath_{n}}\right) \prod_{k=0}^{n-1}\left[Q\left(B_{k+1}\right)\right. \\
& \left.\quad \times P_{k}\left(\alpha_{\jmath_{0}+\cdots+j_{k-1}+1}, \cdots, \alpha_{\jmath_{0}+\cdots+\jmath_{k}}\right)\right] d \alpha_{1} \cdots d \alpha_{J} .
\end{aligned}
$$

If $j_{k}-\leftarrow 0$, the operator valued function $P_{k}$ is equal to

$$
\begin{aligned}
& e^{i \tau\left(t_{k+1}-a_{j_{0}+\cdots+j_{k}}\right)} U_{\varepsilon} e^{i \tau\left(\alpha j_{0}+\cdots+j_{k}-a_{j_{0}}+\cdots+j_{k-1}\right.} \cdots \\
& \cdots U_{\varepsilon} e^{i \tau\left(\alpha_{j_{0}}+\cdots+j_{k-1}+2^{-\alpha} j_{0}+\cdots+j_{k-1}+1\right)} U_{\varepsilon} e^{i \tau \alpha j_{0}+\cdots+j_{k-1}+1}
\end{aligned}
$$

otherwise it is equal to the constant $e^{i \tau t_{k+1}}$, for all $k=0, \cdots, n$.

As mentioned earlier, there exists a $2 \times 2$ unitary matrix $N$ such that for each $g \in L^{2}\left(\mathbb{R}_{+} ; \mathbb{C}^{2}\right), N^{*} e^{i \tau t} N g=f_{t}$ where $f_{t}(x)_{1}=g(x+t)_{1}$ for all $x \geqq 0, f_{t}(x)_{2}=$ $g(x-t)_{2}$ for all $x \geqq t$ and $f_{t}(x)_{2}=0$ for $0 \leqq x \leqq t$. It follows that if $B_{1}, \cdots, B_{n}$ are Borel sets and $t_{1}, \cdots, t_{n}$ are times such that there is no path $\omega \in \Lambda_{J}\left(\mathbb{R}_{+} \times\right.$ $\left.\left(T\left(j_{0}, \cdots, j_{n} ; t_{1}, \cdots, t_{n}\right) \times \Xi_{J}\right)\right) \cap \mathbb{R}_{+}{ }^{[0, \infty)}$ such that $\omega\left(t_{1}\right) \in B_{1}, \cdots, \omega\left(t_{n}\right) \in B_{n}$, then the integrand on the right hand side of (4.5) is identically zero for all values of $\left(\alpha_{1}, \cdots, \alpha_{J}\right) \in T\left(j_{0}, \cdots, j_{n} ; t_{1}, \cdots, t_{n}\right)$ - the integrand may be written as a finite matrix sum over paths belonging $\Lambda_{J}\left(\mathbb{R}_{+} \times\left(T\left(j_{0}, \cdots, j_{n} ; t_{1}, \cdots, t_{n}\right) \times \Xi_{J}\right)\right) \cap$ $\mathbb{R}_{+}{ }^{[0, \infty)}$ and passing through the sets $B_{1}, \cdots, B_{n}$ at times $t_{1}, \cdots, t_{n}$. The representation (4.3) shows that for any set $E$ of the form (1.1) disjoint from $\Lambda_{J}\left(\mathbb{R}_{+} \times \Delta_{J} \times \Xi_{J}\right) \cap \mathbb{R}_{+}^{[0, \infty)}$, the operator $R_{t, J}^{(k, \varepsilon)}(E)$ is the zero operator, and the additivity of $R_{t, J}^{(k, \varepsilon)}$ ensures that $R_{t, J}^{(k, \varepsilon)}(A)=0$ for any set $A$ belonging to the algebra $\alpha\left(R_{t}\right)$ generated by sets of the form (1.1), which is disjoint from $\Lambda_{J}\left(\mathbb{R}_{+} \times \Delta_{J} \times \Xi_{J}\right) \cap \mathbb{R}_{+}{ }^{[0, \infty)}$.

We summarise this discussion in the next statement.

4.1 Lemma. For any set $A \in \alpha\left(\mathscr{R}_{t}\right)$ disjoint from $\Lambda_{J}\left(\mathbb{R}_{+} \times \Delta_{J} \times \Xi_{J}\right) \cap \mathbb{R}_{+}{ }^{[0, \infty)}$, $R_{t, J}^{(k, \varepsilon)}(A)=0$.

The map $\Lambda_{J}: \mathbb{R}_{+} \times \Delta_{J} \times \Xi_{J} \rightarrow C([0, t])$ is uniformly continuous, so it has a unique continuous extension to the closure $\mathbb{R}_{+} \times \bar{J}_{J} \times \Xi_{J}$ of $\mathbb{R}_{+} \times \Delta_{J} \times \Xi_{J}$ in $\mathbb{R}_{+} \times$ $\mathbb{R}^{J} \times \Xi_{J}$. The extension is denoted by the same symbol. Moreover, no new paths are introduced by the continuous extension. It follows that for every compact subset $C$ of $\mathbb{R}_{+}$, the set $\Lambda_{J}\left(C \times \bar{\Delta}_{J} \times \Xi_{J}\right)=\Lambda_{J}\left(C \times \Delta_{J} \times \Xi_{J}\right)$ is is a compact subset of $C([0, t])$. We give $\left.\Lambda_{J}\left(\mathbb{R}_{+} \times \Delta_{J} \times \Xi_{J}\right)\right)$ the relative topology of $C([0, t])$.

Any vector valued Borel measure $m$ on complete separable metric space $X$ is regular, in the sense that for any neighbourhood $U$ of zero in the vector space, and any Borel set $A$ in $X$, there exists a compact subset $K$ of $A$ such that $m(B) \in U$ for all Borel subsets $B$ of $A \backslash K[\mathrm{~S}$, Theorem 9, p 122], [K-K, II.1 Theorem 1]. 
4.2 Lemma. There exists a unique $\mathcal{L}\left(L^{2}\left(\mathbb{R}_{\tau} ; \mathbb{C}^{2}\right)\right)$-valued Borel measure $\bar{R}_{t, J}^{(k, \varepsilon)}: \mathscr{B}\left(\Lambda_{J}\left(\mathbb{R}_{+} \times \Delta_{J} \times \Xi_{J}\right) \cap \mathbb{R}_{+}{ }^{[0, \infty)}\right) \rightarrow \mathcal{L}\left(L^{2}\left(\mathbb{R}_{+} ; \mathbb{C}^{2}\right)\right)$ such that $\bar{R}_{t, J}^{(k, \varepsilon)}\left(A \cap \Lambda_{J}\left(\mathbb{R}_{+} \times\right.\right.$ $\left.\left.\Delta_{J} \times \Xi_{J}\right)\right)=R_{t, J}^{(k, \varepsilon)}(A)$ for all $A \in R_{t}$.

Proof. The spectral measure $Q$ is $\sigma$-additive in $\mathcal{L}\left(L^{2}\left(\mathbb{R}_{+} ; \mathbb{C}^{2}\right)\right)$, so for any function $f \in L^{2}\left(\mathbb{R}_{+} ; \mathbb{C}^{2}\right)$ and $\delta>0$, there exists a compact subset $C_{\delta}$ of $\mathbb{R}_{+}$such that $\left\|R_{t, J}^{(k, \varepsilon)}\left(B \cap\left\{\omega: \omega(0) \in \mathbb{R}_{+} \backslash C_{\delta}\right\}\right) f\right\|_{2}=\left\|R_{t, J}^{(k, \xi)}(B) Q\left(\mathbb{R}_{+} \backslash C_{\delta}\right) f\right\|_{2}<\delta$ for all $B \in \sigma\left(R_{t}\right)$. If $A \in \alpha\left(\mathscr{R}_{t}\right)$ is disjoint from $\Lambda_{J}\left(C_{\hat{o}} \times \bar{J}_{J} \times \Xi_{J}\right) \cap \mathbb{R}_{+}{ }^{[0, \infty)}$, then $A \cap\left\{\omega: \omega(0) \in C_{\hat{o}}\right\}$ is disjoint from $\Lambda_{J}\left(\mathbb{R}_{+} \times \Delta_{J} \times \Xi_{J}\right) \cap \mathbb{R}_{+}{ }^{[0, \infty)}$. It follows from Lemma 4.1 that $\left\|R_{t, J}^{\langle k, \varepsilon)}(B) f\right\|_{2}<\delta$ for all $B \subseteq A$.

For any $g \in L^{2}\left(\mathbb{R}_{+} ; \mathbb{C}^{2}\right),\|g\|_{2} \leqq 1$, the variation $W \mapsto\left|\left(R_{t, J}^{(k, \varepsilon)} f, g\right)\right|(W), W \in$ $\sigma\left(R_{t}\right)$ of the scalar set function $\left(R_{t, J}^{(k, \varepsilon)} f, g\right): W \mapsto\left(R_{t, J}^{(k, \varepsilon)}(W) f, g\right), W \in \sigma\left(\mathscr{R}_{t}\right)$ satisfies the inequality $\left|\left(R_{t, J}^{(k, g)} f, g\right)\right|(W) \leqq 4 \sup _{V \subseteq W}\left\|R_{t, J}^{(k, \varepsilon)}(V) f\right\|_{2}[\mathrm{D}-\mathrm{U}$, I.1.11]. In particular, for any set $A \in \alpha\left(\mathscr{R}_{t}\right)$ disjoint from the compact set $\Lambda_{J}\left(C_{\delta} \times \Delta_{J} \times \Xi_{J}\right)$ $\cap \mathbb{R}_{+}^{[0, \infty)}$, the inequality $\left|\left(R_{t, J}^{(k, \varepsilon)} f, g\right)\right|(A) \leqq 4 \delta$ is valid for all $g \in L^{2}\left(\mathbb{R}_{+} ; \mathbb{C}^{2}\right)$ with $\|g\|_{2} \leqq 1$.

The set function $\left|\left(R_{t, J}^{(k, \varepsilon)} f, g\right)\right|$ is therefore cylindrically concentrated on compact subsets of $C([0, t])[\mathrm{S}, \mathrm{p} 188]$. An application of Prokhorov's theorem [S, Theorem 21, pp 74-75] (which has a direct extension to scalar valued set functions) to the measure $\left(R_{t, J}^{(k, \varepsilon)} f, g\right)$ yields a regular Borel measure on $A_{J}\left(\mathbb{R}_{+}\right.$ $\left.\times \Delta_{J} \times \Xi_{J}\right) \cap \mathbb{R}_{+}{ }^{[0, \infty)}$ for each $g \in L^{2}\left(\mathbb{R}_{+} ; \mathbb{C}^{2}\right)$. The $L^{2}$-bound in $g$ and the OrliczPettis theorem give the required Borel measure $\bar{R}_{t, J}^{(k, \varepsilon)}$. It is unique because the sets $A \cap \Lambda_{J}\left(\mathbb{R}_{+} \times \Delta_{J} \times \Xi_{J}\right), A \in \mathscr{R}_{t}$ generate the Borel $\sigma$-algebra of $\Lambda_{J}\left(\mathbb{R}_{+} \times \Delta_{J} \times\right.$ $\left.\Xi_{J}\right) \cap \mathbb{R}_{+}{ }^{[0, \infty)}[\mathrm{S}$, Lemma $18, \mathrm{p} 108]$.

We give the $F_{\sigma}$-subset $\Gamma=\cup_{J=1}^{\infty} \Lambda_{J}\left(\mathbb{R}_{+} \times \Delta_{J} \times E_{J}\right) \cap \mathbb{R}_{+}{ }^{[0, \infty)}$ of the space $C([0, t])$ the relative topology.

4.3 Theorem. There exists a unique $\mathcal{L}\left(L^{2}\left(\mathbb{R}_{+} ; \mathbb{C}^{2}\right)\right)$-valued Borel measure $\bar{R}_{t}^{(k, s)}: \mathscr{B}(\Gamma) \rightarrow \mathcal{L}\left(L^{2}\left(\mathbb{R}_{+} ; \mathbb{C}^{2}\right)\right)$ such that $\bar{R}_{t}^{(k, s)}(A \cap \Gamma)=R_{t}^{(k, \varepsilon)}(A)$ for all $A \in \sigma\left(\mathscr{R}_{t}\right)$.

Proof. It follows from Lemma 4.2 and equation (4.4) that for any set $A \in$ $\sigma\left(\mathscr{R}_{t}\right)$ disjoint from $\Gamma, R_{t}^{(k, \varepsilon)}(A)=0$. Thus, the set function $A \cap \Gamma \mapsto R_{t}^{(k, \varepsilon)}(A)$, $A \in \sigma\left(R_{t}\right)$ is $\sigma$-additive and it has a uniquely defined extension $\bar{R}_{t}^{(k, \varepsilon)}$ to the Borel subsets of $\Gamma$, such that for all $A \in \mathscr{B}(\Gamma), \bar{R}_{t}^{(k, \varepsilon)}(A)=\sum_{J=0}^{\infty} i^{J} \bar{R}_{t, J}^{(k, \varepsilon)}(A \cap$ $\left.\Lambda_{J}\left(\mathbb{R}_{+} \times \Delta_{J} \times \bar{\Xi}_{J}\right)\right)$.

Denote the space of real valued continuous functions on $[0, \infty)$ by $C([0, \infty))$. It is endowed with the topology of uniform convergence on compact subsets of $[0, \infty)$. It is easily verified that the closure of the set $\cup_{t>0} \cup_{J=1}^{\infty} \Lambda_{J}\left(\mathbb{R}_{+} \times \Delta_{J} \times\right.$ $\left.E_{J}\right) \cap \mathbb{R}_{+}{ }^{[0 \infty}$ in the space $C([0, \infty))$ is the set of all continuous functions $f$ : $[0, \infty) \rightarrow[0, \infty)$ such that $|f(r)-f(s)| \leqq|r-s|$ for all $r, s \geqq 0$. 
Remarks. The argument of Theorem 4.3 provides an alternate proof and a generalisation of the main result of [I-T3]. In the situation considered in [I-T3], for each $J=1,2, \cdots$, the subset of $\Xi_{J}$ consisting of alternating functions is used.

Now let $\Omega$ be the space of those paths $\omega:[0, \infty) \rightarrow \mathbb{R}^{3}$ for which there exists a unit vector $v \in \mathbb{R}^{3}$ such that $\omega=v|\omega|$ and $|\omega| \in \Gamma$. It follows that $\|\boldsymbol{\omega}(s)|-| \omega(t)\| \leqq|t-s|$ for all $t, s \geqq 0$. The space $\Omega$ has the topology of uniform convergence on compact subsets of $[0, \infty)$. By the triangle inequality, the space $\Omega^{\prime}$ of all paths $\omega:[0, \infty) \rightarrow \mathbb{R}^{3}$ such that $|\omega(s)-\omega(t)| \leqq|t-s|$ for all $t, s \geqq 0$ would do as well, in that it contains the former space. Let $X_{s}: \Omega \rightarrow \mathbb{R}^{3}$ denote the evaluation $\operatorname{map} X_{s}(\omega)=\omega(s), \omega \in \Omega, s \geqq 0$.

As defined earlier, $\mathscr{B}_{\mathrm{R}}$ is the sub- $\sigma$-algebra of the Borel sets in $\mathbb{R}^{3}$ consisting of radially symmetric sets. The spectral measure acting on $L^{2} \mathbb{R}^{3} ; \mathbb{C}^{4}$ ) of multiplication by characteristic functions of sets belonging to $\mathscr{B}_{\mathbf{R}}$ is denoted by $Q_{\mathrm{R}}$. For each $t>0$, the semi-algebra of all subsets of $\Omega$ of the form (1.1) with the sets $B_{1}, \cdots, B_{n}$ belonging to $\mathcal{B}_{\mathrm{R}}$ is denoted by $\mathcal{S}_{t}$. Let $S_{D}$ be the unitary group of operators acting on $L^{2}\left(\mathbb{R}^{3} ; C^{4}\right)$ defined by $S_{D}(t)=e^{i D t}$ for all $t \in \mathbb{R}$. The $\left(S_{D}, Q_{\mathbf{R}}, t\right)$-set function is denoted by $M_{t}$.

Because the formula $M_{t}^{(\varepsilon)}\left(\Psi^{-1}(E)\right)=\Sigma_{k, m} \oplus \mathcal{U}_{k, m} * \bar{R}_{t}^{(k, \varepsilon)}(E) \mathcal{U}_{k, m}$ from the proof of Theorem 3.2 works for Borel subsets $E$ of $\Gamma$ as well as cylinder sets, the following result is immediate.

4.4 Theorem. Let $\mathcal{S}_{t}$ be the semi-algebra of elementary events in $\Omega$. For each $t>0$ and $\varepsilon>0$, there exists a unique operator valued measure $M_{t}^{(\varepsilon)}: \sigma\left(\mathcal{S}_{t}\right) \rightarrow$ $\mathcal{L}\left(L^{2}\left(\mathbb{R}^{3} ; \mathbb{C}^{4}\right)\right)$ such that the restriction of $M_{t}^{(\varepsilon)}$ to $\mathcal{S}_{t}$ is the $\left(S_{D_{\varepsilon}}, Q_{\mathbf{R}}, t\right)$-set function.

The operator valued set function $M_{t}$ is the limit of the $\sigma$-additive valued measures $M_{t}^{(\varepsilon)}$ in the sense that, as $\varepsilon \rightarrow 0^{+}, M_{t}^{(\varepsilon)}(A) \rightarrow M_{t}(A)$ in the strong operator topology for each $A$ belonging to the algebra $\alpha\left(\mathcal{S}_{t}\right)$ generated by the collection $\mathcal{S}_{t}$ of elementary events.

\section{§5. Integration with Respect to $M_{t}$ and $\left\langle M_{t}^{(\varepsilon)}\right\rangle_{\varepsilon}>0$}

The operator valued set function $M_{t}$ is the strong operator limit of the family $\left\langle M_{t}^{(\varepsilon)}\right\rangle_{\varepsilon}>0$ of operator valued measures on the algebra $\alpha\left(\mathcal{S}_{t}\right)$. It is therefore natural to view integration with respect to $M_{t}$ as being controlled by the measures $\left.\left\langle M_{t}^{(\varepsilon)}\right\rangle_{\varepsilon}\right\rangle_{0}$; this is the viewpoint adopted in [J1] in the context of the Schrödinger equation. What results in the present context is a quasi-complete space $L^{1}\left(M_{t}^{+}, M_{t}\right)$ of functions integrable with respect to $M_{t}$, relative to the family $\left\langle M_{t}^{(\varepsilon)}\right\rangle_{\varepsilon}>_{0}$ of operator valued measures. The payoff is in the next section, whose results are proved as if $M_{t}$ were actually a $\sigma$-additive operator valued measure. 
The few facts and definitions we need concerning integration with respect to vector measures are given in $[\mathrm{K}-\mathrm{K}]$. For example, in the present context, all bounded measurable functions are integrable [K-K, II.3 Lemma 1]. A set $N$ is a null set with respect to a vector measure $m$ with values in a locally convex space $X$, if and only if $N$ is null with respect to the variation $\left|\left\langle m, x^{\prime}\right\rangle\right|$ of the scalar measure $\left\langle m, x^{\prime}\right\rangle$ for each $x^{\prime}$ belonging to the continuous dual $X^{\prime}$ of $X[\mathrm{~K}-\mathrm{K}, \mathrm{p} 22]$. The usual terminology concerning properties occurring "almost everywhere" with respect to $m$ then has a meaning.

Throughout this section $t>0$ is a fixed number. An $\mathcal{S}_{t}$-simple function $s$ is a finite linear combination $\sum_{j=1}^{n} c_{j} \chi_{A_{j}}, c_{\jmath} \in \mathbb{C}$ of characteristic functions of sets $A_{\jmath} \in \mathcal{S}_{t}, j=1, \cdots, n, n=1,2, \cdots$. The set function $s M_{t}: \mathcal{S}_{t} \rightarrow \mathcal{L}\left(L^{2}\left(\mathbb{R}^{3} ; \mathbb{C}^{4}\right)\right)$ is defined by $s M_{t}(A)=\sum_{j=1}^{n} c_{j} M_{t}\left(A \cap A_{\jmath}\right)$ for every $A \in \mathcal{S}_{t}$. Set $M_{t}(s)=s M_{t}(\Omega)$.

Let $f: \Omega \rightarrow \mathbb{C}$ be a $\sigma\left(S_{t}\right)$-measurable function. The collection of all functions $g$ such that the set $\{\omega \in \Omega: g(\omega) \pm f(\omega)\}$ is $M_{t}^{(\varepsilon)}$-null for every $\varepsilon>0$ is denoted by $[f]_{+}$. Let $\left.L^{1}\left(\left\langle M_{t}^{(\varepsilon)}\right\rangle_{s}\right\rangle_{0}\right)$ denote the collection of all equivalence classes $[f]_{+}$ of $\sigma\left(\mathcal{S}_{t}\right)$-measurable functions $f$ which are integrable with respect to $M_{t}^{(\varepsilon)}$ for every $s>0$. Then $\left.L^{1}\left(\left\langle M_{t}^{(s)}\right\rangle_{\varepsilon}\right\rangle_{0}\right)$ has the vector space structure defined by $[f]_{+}$ $+[g]_{+}=[f+g]_{+}, a[f]_{+}=[a f]_{+}$for all $\left.[f]_{+},[g]_{+} \in L^{1}\left(\left\langle M_{t}^{(\varepsilon)}\right\rangle_{\varepsilon}\right\rangle_{0}\right)$ and $a \in \mathbb{C}$. For every $\varepsilon>0$ and $\phi \in L^{2}\left(\mathbb{R}^{3} ; \mathbb{C}^{4}\right)$, let $\Gamma_{t, q, \varepsilon}$ be the family of measures $\left\{\left|\left(M_{t}^{(\varepsilon)} \phi, \phi\right)\right|:\|\phi\|_{2} \leqq 1\right\}$. The space $L^{1}\left(\left\langle M_{t}^{(\varepsilon)}\right\rangle_{\varepsilon}>0\right)$ is given the locally convex topology defined by the family $\left\{p_{t, \phi, \varepsilon}: \varepsilon>0, \phi \in L^{2}\left(\mathbb{R}^{3} ; \mathbb{C}^{4}\right)\right\}$ of seminorms, defined for each $\varepsilon>0$ and $\phi \in L^{2}\left(\mathbb{R}^{3} ; \mathbb{C}^{4}\right)$ by $p_{t, \phi, \varepsilon}\left([f]_{+}\right)=\sup \left\{\int_{\Sigma}|f| d \mu: \mu \in \Gamma_{t, \phi, \varepsilon}\right\}$ for each $\left.[f]_{+} \in L^{1}\left(\left\langle M_{t}^{(c)}\right\rangle_{\varepsilon}\right\rangle 0\right)$.

A subset $A$ of a Borel set $N$ for which $\left[\chi_{N}\right]_{+}=0$ is said to be $M_{t}^{+}$-null. A property which obtains off an $M_{t}^{+}$-null set is said to be satisfied $M_{t}^{+}$-almost everywhere (briefly, $M_{t}^{+}$-a.e.). A function $f$ such that $\left.[f]_{+} \in L^{1}\left(\left\langle M_{t}^{(\varepsilon)}\right\rangle_{\varepsilon}\right\rangle_{0}\right)$ is said to be $M_{t}^{+}$-integrable. The space $\left.L^{1}\left(\left\langle M_{t}^{(s)}\right\rangle_{\varepsilon}\right\rangle_{0}\right)$ will sometimes be written, more briefly as $L^{1}\left(M_{t}^{+}\right)$.

5.1 Proposition. The locally convex space $L^{1}\left(M_{t}^{+}\right)$is quasi-complete.

Proof. A small modification of the proof of [K-K, Theorem IV.7.3] shows that it is enough to exhibit a finite measure $\mu: \sigma\left(\mathcal{S}_{t}\right) \rightarrow[0, \infty)$ such that $M_{t}^{(\varepsilon)}$ is absolutely continuous with respect to $\mu$ for each $\varepsilon>0$, for then the associated measure algebra is complete. The conclusion of quasi-completeness rather than completeness is related to the observation that the space $\mathcal{L}\left(L^{2}\left(\mathbb{R}^{3} ; \mathbb{C}^{4}\right)\right)$ of bounded linear operators acting on $L^{2}\left(\mathbb{R}^{3} ; \mathbb{C}^{4}\right)$ is only quasi-complete.

First we construct a related operator valued measure acting on $L^{1}\left(\mathbb{R}_{+} ; \mathbb{C}^{2}\right)$. Let $T$ denote the operator $T_{+}$defined in section two. Then for the matrix $N=1 / \sqrt{2}\left(\begin{array}{ll}1 & i \\ i & 1\end{array}\right)$, the equality $N^{*} \tau N=T$ obtains and we have $N * U_{k, \varepsilon}(r) N=$ $\left(\begin{array}{cc}0 & i m-k / r \\ -i m-k / r & 0\end{array}\right)$ for $r>\varepsilon$ and $N * U_{k, s}(r) N=\left(\begin{array}{c}0 \\ -i m-k / \varepsilon\end{array}{ }^{i m-k / \varepsilon}\right)$ for $0<$ 
$r \leqq \varepsilon . \quad$ Let $\sigma_{1}=\left(\begin{array}{ll}0 & 1 \\ 1 & 0\end{array}\right)$ and set $W_{0}^{(\varepsilon)}(s)=e^{i T s}$ and

$$
W_{j}^{(s)}(s)=(m+|k| / \varepsilon)^{j} \int_{0}^{s} \cdots \int_{0}^{a_{2}} e^{T\left(s-a_{j}\right)} \sigma_{1} \cdots e^{T\left(a_{2}-\alpha_{1}\right)} \sigma_{1} e^{T a_{1}} d \alpha_{1} \cdots d \alpha_{j}
$$

for all $s>0, j=1,2, \cdots$ and $k \in \mathbb{Z} \backslash\{0\}$. Then $\left\|W_{j}^{(\varepsilon)}(s)\right\| \leqq(m+|k| / \varepsilon)^{j} s^{j} / j !$. If we set

$$
\tilde{R}_{t, J}^{(k, \varepsilon)}(E)=\sum_{j_{0}+\cdots+j_{n}=J} W_{j_{n}}^{(\varepsilon)}\left(t-t_{n}\right) Q\left(B_{n}\right) W_{j_{n-1}}^{(\varepsilon)}\left(t_{n}-t_{n-1}\right) \cdots W_{j_{1}}^{(\varepsilon)}\left(t_{2}-t_{1}\right) Q\left(B_{1}\right) W_{j_{0}}^{(\varepsilon)}\left(t_{1}\right)
$$

for each set $E$ of the form (1.1) and $J=0,1,2, \cdots$, then it is a simple matter to check that the sum $\tilde{R}_{t}^{(k, \varepsilon)}=\sum_{J=0}^{\infty} \tilde{R}_{t, J}^{(k, \varepsilon)}$ defines an $\mathcal{L}\left(L^{1}\left(\mathbb{R}_{+} ; \mathbb{C}^{2}\right)\right)$-valued Borel measure, as in section four. Moreover, if $\phi \in L^{1}\left(\mathbb{R}_{+} ; \mathbb{C}^{2}\right)$ has real, non-negative components, then $0 \leqq\left(\tilde{R}_{t}^{(k \varepsilon)} \phi, \phi\right) \leqq\left(\tilde{R}_{t}^{(k, \delta)} \phi, \phi\right)$ for all $0<\delta \leqq \varepsilon$ and if $N^{*} \phi$ has non-negative components, then $\left|\left(\bar{R}_{t}^{(k, \varepsilon)} \phi, \phi\right)\right|(A) \leqq\left(\tilde{R}_{t}^{(k, \varepsilon)} N^{*} \phi, N^{*} \phi\right)(A)$ for all $A \in$ $\mathscr{B}(\Gamma)$.

In the notation of section four, $M_{l}^{(\varepsilon)}\left(\Psi^{-1}(E)\right)=\sum_{k, m} \oplus \mathcal{U}_{k, m} * \bar{R}_{t}^{(k, \varepsilon)}(E) \mathcal{U}_{k, m}$ for all $E \in \sigma\left(\mathscr{R}_{t}\right)$. Let $\phi_{k, m, j}, j=1,2, \cdots$ be an orthonormal basis of the orthogonal subspaces $\mathscr{H}_{k, m}$ which reduce $D$, introduced in section two. Then by the preceding observations, each of the measures $M_{t}^{(\varepsilon)}, \varepsilon>0$ is absolutely continuous with respect to the finite measure $\mu$ with

$$
\mu\left(\Psi^{-1}(E)\right)=\sum_{j, k} \sum_{l, m, n} a_{j, k, l, m, n}\left|\left(\widetilde{R}_{t}^{(k, 1 / n)}(E) \bigcup_{k, m} \phi_{k, m, j}, \mathcal{U}_{k, m} \phi_{k, m, l}\right)\right|,
$$

$$
\text { for all } E \in \sigma\left(\mathscr{R}_{t}\right) \text {, }
$$

normalised with

$$
a_{j, k, l, m, n}=\left[\left|\left(\widetilde{R}_{t}^{(k, 1 / n)} \bigcup_{k, m} \phi_{k, m, j}, \mathcal{U}_{k, m} \phi_{k, m, l}\right)\right|(\Gamma)\right]^{-1} 2^{-(j+k+l+m+n)} .
$$

5.2 Lemma. $M_{t}^{(\varepsilon)}(A) \rightarrow M_{t}(A)$ in the strong operator topology as $\varepsilon \rightarrow 0^{+}$, for each $A \in \mathcal{S}_{t}$.

Proof. The limit $\lim _{\varepsilon \rightarrow 0} e^{i D_{\varepsilon} s}=e^{i D s}$ exists in the strong operator topology of $\mathcal{L}\left(L^{2}\left(\mathbb{R}^{3} ; \mathbb{C}^{4}\right)\right)$, uniformly for $s$ in compact subsets of $[0, \infty)$, so the result follows from formula (1.2) by the Banach-Steinhaus theorem.

If $s$ is any $\mathcal{S}_{t}$-simple function, then $s M_{t}^{(\varepsilon)}(A) \rightarrow s M_{t}(A)$ in the strong operator topology as $\varepsilon \rightarrow 0^{+}$for every $A \in \mathcal{S}_{t}$. It follows that the map $[s]_{+} \mapsto s M_{t}$, defined for all $\mathcal{S}_{t}$-simple function $s$ is well-defined. Then the subspace $\operatorname{sim}\left[\mathcal{S}_{t}\right]=\left\{[s]_{+}\right.$: $s$ is $\mathcal{S}_{t}$-simple\} of $L^{1}\left(M_{t}^{+}\right)$may be endowed with the locally convex topology $\tau\left(M_{t}\right)$ defined by the family $\left\{q_{t, \phi, A}: A \in \mathcal{S}_{t}, \phi \in L^{2}\left(\mathbb{R}^{3} ; \mathbb{C}^{4}\right)\right\}$ of seminorms with $q_{t, \phi, A}\left([s]_{+}\right)=\sup _{0<\varepsilon \leq 1}\left|\left(\left(s M_{t}^{(\varepsilon)}\right)(A) \phi, \phi\right)\right|,[s]_{+} \in \operatorname{sim}\left[\mathcal{S}_{t}\right]$, for each $A \in \mathcal{S}_{t}$ and $\phi \in$ $L^{2}\left(\mathbb{R}^{3} ; \mathbb{C}^{4}\right)$.

The next assertion shows that the identity map on $\operatorname{sim}\left[\mathcal{S}_{t}\right]$ is a closable linear map from $L^{1}\left(M_{t}^{+}\right)$to the completion $\operatorname{sim}\left[\mathcal{S}_{t}\right]$ of $\operatorname{sim}\left[\mathcal{S}_{t}\right]$ with respect to the topology $\tau\left(M_{t}\right)$. 
5.3 Lemma. If $\left\langle\left[s_{l}\right]_{+}\right\rangle_{l \in I}$ is a net in $\left[\mathcal{S}_{t}\right]$ converging to zero in $L^{1}\left(M_{t}^{+}\right)$ and $\left\langle\left[s_{l}\right]_{+}\right\rangle_{l \in I}$ is a Cauchy net in $\tau\left(M_{t}\right)$, then $\lim _{l \in I}\left[s_{l}\right]_{+}=0$ in $\tau\left(M_{t}\right)$.

Proof. That $\left\langle\left[s_{l}\right]_{\tau}\right\rangle_{l \in I}$ is a Cauchy net in $\tau\left(M_{t}\right)$, means that the net $\left\langle\left(\left(s_{l} M_{t}^{(\varepsilon)}\right)(A) \phi, \phi\right)\right\rangle_{l \in I}$ converges uniformly for all $0<\varepsilon \leqq 1$, for each $A \in \mathcal{S}_{t}$ and $\phi \in L^{2}\left(\mathbb{R}^{3} ; \mathbb{C}^{4}\right)$. But the limit must be zero, because $\left\langle\left[s_{l}\right]_{+}\right\rangle_{l \in I}$ converges to 0 in $L^{1}\left(M_{t}^{+}\right)$, so $\lim _{l \in I}\left[s_{l}\right]_{+}=0$ in $\tau\left(M_{t}\right)$.

It follows from Lemma 5.3 that the linear space of all bounded Cauchy nets of elements of $\operatorname{sim}\left[\mathcal{S}_{t}\right]$ in the locally convex topology determined by the family $\mathscr{Q}_{t}=\left\{p_{t, \phi, \varepsilon}+q_{t, \phi, A}: \varepsilon>0, \phi \in L^{2}\left(\mathbb{R}^{3} ; \mathbb{C}^{4}\right), A \in \mathcal{S}_{t}\right\}$ of seminorms can be identified with a subspace $L^{1}\left(M_{t}^{+}, M_{t}\right)$ of $L^{1}\left(M_{t}^{+}\right)$. The locally convex space $L^{1}\left(M_{t}^{+}, M_{t}\right)$ has the topology determined by the family of seminorms $\mathscr{Q}_{t}$. We note here an obvious consequence.

5.4 Proposition. The locally convex space $L^{1}\left(M_{t}^{+}, M_{t}\right)$ is quasi-complete.

5.5 Lemma. For each $[f]_{+} \in L^{1}\left(M_{t}^{+}, M_{t}\right)$ and $A \in \mathcal{S}_{t}$, the limits $f M_{t}(A)=$ $\lim _{\varepsilon \rightarrow 0} f M_{t}^{(\varepsilon)}(A)$ and $\left(f M_{t}\right)^{*}(A)=\lim _{\varepsilon \rightarrow 0}\left[f M_{t}^{(\varepsilon)}(A)\right]^{*}$ exist in the weak operator topology. Moreover, $f M_{t}: \mathcal{S}_{t} \rightarrow \mathcal{L}\left(L^{2}\left(\mathbb{R}^{3} ; \mathbb{C}^{4}\right)\right)$ and $\left(f M_{t}\right)^{*}: \mathcal{S}_{t} \rightarrow \mathcal{L}\left(L^{2}\left(\mathbb{R}^{3} ; \mathbb{C}^{4}\right)\right)$ are additive set functions.

A function $f: \Omega \rightarrow \mathbb{C}$ such that $[f]_{+} \in L^{1}\left(M_{t}^{+}, M_{t}\right)$ is said to be $M_{t}^{+}-M_{t^{-}}$ integrable. The conventional notation $\int_{A} f(\omega) d M_{t}(\omega)$ is sometimes used for the

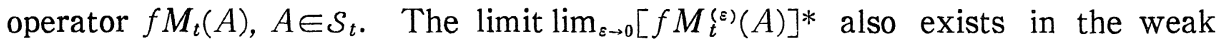
operator topology, so we write this as $\bar{f} M_{t}^{*}(A)=\int_{A} \overline{f(\omega)} d M_{t}^{*}(\omega)$.

The next section is devoted to a class of $M_{t}^{+}-M_{t}$-integrable functions. For the moment, we have the following

5.6 Proposition. Let $F_{1}, \cdots, F_{n}$ be radially symmetric, bounded, Borel measurable functions on $\mathbb{R}^{3}$. Let $0 \leqq t_{1}<\cdots<t_{n} \leqq t$. Then the function $f: \omega \mapsto F_{1}\left(\omega\left(t_{1}\right)\right)$ $\cdots F_{n}\left(\omega\left(t_{n}\right)\right), \omega \in \Omega \quad$ is $M_{t}^{+}-M_{t}$-integrable and $\int_{\Omega} f d M_{t}=e^{i D\left(t-t_{n}\right)} Q_{\mathbf{R}}\left(F_{n}\right) \cdots$ $e^{i D\left(t_{2}-t_{1}\right)} Q_{\mathbf{R}}\left(F_{1}\right) e^{i D t_{1}}$.

Proof. Suppose that $F$ is a radially symmetric bounded Borel measurable function. Then there exists radially symmetric simple functions $s_{n}, n=1,2, \cdots$ on $\mathbb{R}^{3}$ such that $s_{n} \rightarrow F$ uniformly on $\mathbb{R}^{3}$ as $n \rightarrow \infty$. Then $Q_{\mathbf{R}}\left(s_{n}\right) \rightarrow Q(F)$ in the strong operator topology and uniformly on precompact sets as $n \rightarrow \infty$. In particular, as $n \rightarrow \infty, Q_{\mathbf{R}}\left(s_{n}\right) e^{i D_{\varepsilon^{s}}} \phi \rightarrow Q(F) e^{i D_{\varepsilon^{s}} \phi}$ uniformly for $0<\varepsilon \leqq 1$ and for $\phi$ ranging over a precompact subset of $L^{2}\left(\mathbb{R}^{3} ; \mathbb{C}^{4}\right)$. The conclusion follows by induction. 
The following convergence results are immediate consequences of the definitions and the corresponding convergence results for vector measures $[\mathrm{K}-\mathrm{K}$, II.4 Theorem 2].

5.7 Proposition. Let $f_{n}, n=1,2, \cdots$ be $M_{t}^{+}-M_{t}$-integrable functions for which there exists a function $g \geqq 0$ with $[g]_{+} \in L^{1}\left(M_{t}^{+}\right)$and $\left|f_{n}\right| \leqq g M_{t}^{+}-a$.e. for all $n=1,2, \cdots$. If $f_{n}, n=1,2, \cdots$ converges $M_{t}^{+}$-a.e. to a function $f$, and for each $A \in \mathcal{S}_{t}$, the operators $f_{n} M_{t}^{(\varepsilon)}(A), n=1,2, \cdots$ converge in the weak operator topology, uniformly for $\varepsilon>0$, then $f$ is $M_{t}^{+}-M_{t}$-integrable and $\int_{A} f d M_{t}=$ $\lim _{n \rightarrow \infty} \int_{A} f_{n} d M_{t}$ in the weak operator topology for each $A \in \mathcal{S}_{t}$.

5.8 Proposition. Let $f_{n}, n=1,2, \cdots$ be a sequence of $M_{t}^{+}-M_{t}$-integrable functions, increasing $M_{t}^{+}$-a.e., for which $\sup _{n}\left|\left(M_{t}^{(\varepsilon)} \phi, \phi\right)\right|\left(f_{n}\right)<\infty$ for every $\phi \in$ $L^{2}\left(\mathbb{R}^{3} ; \mathbb{C}^{4}\right)$ and $\varepsilon>0$. If $f_{n}, n=1,2, \cdots$ converges $M_{t}^{+}-a$.e. to a function $f$, and for each $A \in \mathcal{S}_{t}$, the operators $f_{n} M_{t}^{(\varepsilon)}(A), n=1,2, \cdots$ converge in the weak operator topology, unisormly for $\varepsilon>0$, then $f$ is $M_{t}^{+}-M_{t}$-integrable and $\int_{A} f d M_{t}=$ $\lim _{n \rightarrow \infty} \int_{A} f_{n} d M_{\iota}$ in the weak operator topology for each $A \in \mathcal{S}_{t}$.

Remark. It is unreasonable to expect that the seminorms $q_{\dot{\phi} . A}$ could be replaced by, say, $q_{\varphi, A^{\prime}}\left([s]_{+}\right)=\left\|\left(s M_{t}\right)(A) \phi\right\|,[s]_{+} \in \operatorname{sim}\left[\mathcal{S}_{t}\right]$, for each $A \in \mathcal{S}_{t}$ and $\phi \in L^{2}\left(\mathbb{R}^{3} ; \mathbb{C}^{4}\right)$, that is, setwise convergence on $\mathcal{S}_{t}$, because $e^{i D t}$ is convolution with a distribution of order one. A similar phenomenon occurs with the Schrödinger equation, although the relevant kernel $x \mapsto(2 \pi i t)^{-3 / 2} e^{i|x|^{2 / 2 t}}, x \in \mathbb{R}^{3}$ is a distribution of order zero.

\section{§6. Path Integral Representation of the Dynamical Group}

Now that the notion of integration with respect to the operator valued set functions $M_{t}, t>0$ is established, the representation of the dynamical group for the Dirac equation with a spherically symmetric potential follows in a straightforward way from dominated convergence, Proposition 5.7.

A function $V: \mathbb{R}^{3} \rightarrow \mathbb{R}$ is said to be spherically symmetric if there exists a function $q:[0, \infty) \rightarrow \mathbb{R}$ such that $V(x)=q(|x|)$ for all $x \in \mathbb{R}^{3}$.

By $[\mathrm{K}, \mathrm{V} .4 .3]$, for any bounded function $V: \mathbb{R}^{3} \rightarrow \mathbb{R}$, the operator $D-V$ is selfadjoint on the same domain for which $D$ is selfadjoint (the Sobolev space $\left.\left[H^{1}\left(\mathbb{R}^{3}\right)\right]^{4}\right)$.

6.1 Lemma. Let $V: \mathbb{R}^{3} \rightarrow \mathbb{R}$ be a bounded, continuous, spherically symmetric function. Then the function $\omega \mapsto \exp \left[-i \int_{0}^{t} V(\omega(s)) d s\right], \omega \in \Omega$ is $M_{t}^{+}$-M $M_{t}$-integrable.

Proof. If $V$ is bounded and continuous, then for each $\omega \in \Omega$, the function 
$s \mapsto V(\omega(s)), 0 \leqq s \leqq t$ is continuous, so the Riemann sums $\sum_{j=1}^{n} V(\omega(t j / n)) t / n, n=$ $1,2, \cdots$ converge to $\int_{0}^{t} V(\omega(s)) d s$, so by dominated convergence, $\Pi_{j=1}^{n} \exp [-i V$ 。 $\left.X_{t j / n} t / n\right] \rightarrow \exp \left[-i \int_{0}^{t} V \circ X_{s} d s\right]$ in $L^{1}\left(M_{t}^{(s)}\right)$ for each $\varepsilon>0$.

It is enough to show that as $n \rightarrow \infty$, the operators $\int_{\Omega} \Pi_{j=1}^{n} \exp \left[-i V \circ X_{t j i n} t / n\right]$ $d M_{t}^{(\varepsilon)}, n=1,2, \cdots$ converge in the strong operator topology of $\mathcal{L}\left(L^{2}\left(\mathbb{R}^{3} ; \mathbb{C}^{4}\right)\right)$, uniformly for $0<\varepsilon \leqq 1$, for then, the same statement holds for the operators $\int_{E} \Pi_{j=1}^{n} \exp \left[-i V \circ X_{t j / n} t / n\right] d M_{t}^{(\varepsilon)}, n=1,2, \cdots, E \in \mathcal{S}_{t}$ by virtue of formula (1.2). It then follows from the definition of the family $\mathscr{P}_{t}$ of seminorms in section five, that $\Pi_{j=1}^{n} \exp \left[-i V \circ X_{t \jmath / n} t / n\right]$ converges in $L^{1}\left(M_{t}^{+}, M_{t}\right)$ to $\exp \left[-i \int_{0}^{t} V \circ X_{s} d s\right]$.

Now $M_{t}^{(\varepsilon)}\left(\Psi^{-1}(E)\right)=\sum_{k, m} \oplus \mathcal{U}_{k, m} * R_{t}^{(k, \varepsilon)}(E) \bigcup_{k, m}, E \in \sigma\left(\mathscr{R}_{t}\right)$ for the map $\Psi:$ $\Omega \rightarrow \mathbb{R}_{+}{ }^{[0, \infty)}$ defined by $(\Psi(\omega))(s)=|\omega(s)|$ for all $s \geqq 0$ and all $\omega \in \Omega$, so by virtue of the spherical symmetry of $V(x)=q(|x|), x \in \mathbb{R}^{3}$, it is enough to show that for each $k \in \mathbb{Z} \backslash\{0\}$, the operators $\int_{\Gamma} \Pi_{\jmath=1}^{n} \exp \left[-i q \circ X_{t \jmath / n} t / n\right] d R_{t}^{(k, \varepsilon)}=$ $\left[e^{-i q t / n} e^{i z_{k}, \varepsilon t / n}\right]^{n}, n=1,2, \cdots$ converge in the strong operator topology of $\mathcal{L}\left(L^{2}\left(\mathbb{R}_{+} ; \mathbb{C}^{2}\right)\right)$, uniformly for $0<\varepsilon \leqq 1$; this follows from the conclusion of Lemma 2.7 .

6.2 Lemma. Let $V: \mathbb{R}^{3} \rightarrow \mathbb{C}$ be a bounded, spherically symmetric Borel measurable function. Then for every $\omega \in \Omega$ and $t>0$, the function $s \mapsto V(\omega(s))$, $0 \leqq s \leqq t$ is integrable, and the function $\omega \mapsto \exp \left[ \pm i \int_{0}^{t} V(\omega(s)) d s\right], \omega \in \Omega$ is $M_{t}^{+}-M_{t^{-}}$ integrable. Furthermore, the equalities $e^{i(D-V) t}=\int_{\Omega} \exp \left[-i \int_{0}^{t} V(\omega(s)) d s\right] d M_{t}(\omega)$ and $e^{-i(D-V) t}=\int_{\Omega} \exp \left[i \int_{0}^{t} V(\omega(s)) d s\right] d M_{t}^{*}(\boldsymbol{\omega})$ hold for all $t>0$.

Proof. The function $s \mapsto V(\omega(s)), 0 \leqq s \leqq t$ is bounded and Borel measurable, so it is integrable for every $\omega \in \Omega$ and $t>0$. It follows from the assumption of spherical symmetry that the function $\left.\omega \mapsto \exp \left[ \pm i \int_{0}^{t} V(\omega)(s)\right) d s\right], \omega \in \Omega$ is bounded and $\sigma\left(\mathcal{S}_{t}\right)$-measurable, so it is $M_{t}^{+}$-integrable [K-K, II.3 Lemma 1]. It is $M_{t}^{+}$$M_{t}$-integrable by virtue of Lemma 2.7 .

The conclusion follows from Lemma 2.7, however, the following proof is more integration theoretic, see $[\mathrm{Si}, \mathrm{p} \mathrm{50]}$ for the case of Wiener measure. Let $\varepsilon>0$. Suppose that $f, g \in L^{2}\left(\mathbb{R}^{3} ; \mathbb{C}^{4}\right)$. For every $0<s \leqq t$ and $t>0$, let

$$
u(s)=\int_{\Omega} \exp \left[-i \int_{0}^{s} V(\boldsymbol{\omega}(r)) d r\right] d\left(M_{s}^{(\varepsilon)} f\right)(\boldsymbol{\omega}) .
$$

Denote the Lebesgue measure on $[0, t]$ by $\lambda$. The function

$$
(\omega, s) \mapsto V(\omega(s)) \exp \left[-i \int_{0}^{s} V(\boldsymbol{\omega}(r)) d r\right], \quad \omega \in \Omega, 0 \leqq s \leqq t
$$


is bounded and $\sigma\left(\mathcal{S}_{t}\right) \otimes \mathscr{B}([0, t])$-measurable, so it is integrable with respect to the product $\left(M_{s}^{(\varepsilon)} f, g\right) \otimes \lambda$ of the scalar measure $\left(M_{s}^{(s)} f, g\right)$ with $\lambda$. Then according to Fubini's theorem

$$
\begin{aligned}
\int_{\Omega \sim[0 t]}-i V(\omega(s)) \exp \left[-i \int_{0}^{s} V(\omega(r)) d r\right] d\left(\left(M_{t}^{(s)} f, g\right) \otimes \lambda\right)(\omega, s) \\
\quad=\int_{\Omega,[0, \iota]} \frac{d}{d s} \exp \left[-i \int_{0}^{s} V(\omega(r)) d r\right] d\left(\left(M_{t}^{(\varepsilon)} f, g\right) \otimes \lambda\right)(\omega, s) \\
\quad=(u(t), g)-\left(M_{t}^{(\varepsilon)} f, g\right)(\Omega)=(u(t), g)-\left(S_{D_{\varepsilon}}(t) f, g\right) \\
\quad=-i \int_{0}^{t}\left(S_{D_{\varepsilon}}(t-s) Q_{\mathbf{R}}(V) u(s), g\right) d s .
\end{aligned}
$$

From this equation, we deduce that $t \longmapsto u(t), t>0$ is continuous in $L^{2}\left(\mathbb{R}^{3} ; \mathbb{C}^{1}\right)$ and it has the same "Dyson series" expansion as $e^{\imath\left(D_{\varepsilon}-V\right) t} f$, see, for example, [K, Theorem IX.2.1]. The analogous argument holds for $e^{-\imath\left(D_{\varepsilon}-V\right) t}$. In the limit,

$$
\begin{aligned}
e^{\imath(D-V) \iota} & \left.=\lim _{\varepsilon \rightarrow 0} e^{\imath\left(D_{\varepsilon}-V\right) t}=\lim _{\varepsilon \rightarrow 0} \int_{\Omega} \exp \left[-i \int_{0}^{t} V(\boldsymbol{\omega}(s)) d s\right] d M_{t}^{(\varepsilon)}(\boldsymbol{\omega})\right) \\
& =\int_{\Omega} \exp \left[-i \int_{0}^{\iota} V(\boldsymbol{\omega}(s)) d s\right] d M_{\iota}(\boldsymbol{\omega}) .
\end{aligned}
$$

6.3 Theorem. Let $V: \mathbb{R}^{3} \rightarrow \mathbb{C}$ be a function such that $V(x)=q(|x|), x \in \mathbb{R}^{3}$ for some locally integrable function $q:[0, \infty) \rightarrow \mathbb{R}$ which is also locally square integrable on $(0, \infty)$. Then for every $t>0$, the function $s \mapsto V(\omega(s)), 0 \leqq s \leqq t$ is integrable for all $\omega \in \Omega$ and the function $\omega \mapsto \exp \left[-i \int_{0}^{t} V(\omega(s)) d s\right], \omega \in \Omega$ is $M_{t}^{+}$$M_{t}$-integrable.

Futhermore, $D-V$ is essentially self-adjoint on $C_{c}^{\infty}\left(\mathbb{R}^{3}, \mathbb{C}^{1}\right)$ and

$$
e^{\imath(D-V) \iota}=\int_{\Omega} \exp \left[-i \int_{0}^{t} V(\omega(s)) d s\right] d M_{t}(\boldsymbol{\omega}), \quad \text { for all } t>0 .
$$

Proof. Let $V_{n}(x)=V(x)$ for all $x \in \mathbb{R}^{3}$ such that $|V(x)| \leqq n$, and let $V_{n}(x)$ $=0$ otherwise. Then by dominated convergence, as $n \rightarrow \infty, \exp \left[-i \int_{0}^{t} V_{n}(\omega(s)) d s\right]$ $\rightarrow \exp \left[-i \int_{0}^{\iota} V(\omega(s)) d s\right]$ for $M_{t}^{+}$-almost all $\omega \in \Omega$. On an appeal to Lemma 6.2, we see that the function $f_{n}: \omega \mapsto \exp \left[-i \int_{0}^{\iota} V_{n}(\omega(s)) d s\right], \omega \in \Omega$ is $M_{t}^{+}-M_{l^{-}}$ integrable, and $e^{i\left(D-r_{n}\right) t}=\int_{\Omega} f_{n}(\omega) d M_{t}(\omega)$ for all $t>0$.

As mentioned in Lemma 2.5, $\tau_{k}-q$ is essentially selfadjoint on $C_{c}^{\infty}\left((0, \infty), \mathbb{C}^{2}\right)$ for each $k \in \mathbb{Z} \backslash\{0\}$, so $D-V$ is essentially self-adjoint on $C_{c}^{\infty}\left(\mathbb{R}^{3}, \mathbb{C}^{4}\right)$. Corollary 2.6 and dominated convergence, Proposition 5.7, ensure that the functions $f_{n}$, $n=1,2, \cdots$ converge in $L^{1}\left(M_{t}^{\tau}, M_{t}\right)$ to the function $f: \omega \rightarrow \exp \left[-i \int_{0}^{t} V(\omega(s)) d s\right]$, $\omega \in \Omega$. In particular, the operators $\int_{\Omega} f_{n}(\boldsymbol{\omega}) d M_{t}(\boldsymbol{\omega})=e^{\imath\left(D-V_{n}\right) t}, n=1,2, \cdots$ converge in the strong operator topology to $\int_{\Omega} f(\boldsymbol{\omega}) d M_{t}(\boldsymbol{\omega})=e^{\imath(D-V) t}$. 
The treatment of the Coulomb potential requires a multiplicative functional other than the Kac functional $\omega \mapsto \exp \left[-i \int_{0}^{t} V(\omega(s)) d s\right]$, because there is a non$M_{t}^{+}$-negligible set of paths which hit the origin.

In view of the observation of $[\mathrm{I}-\mathrm{T}]$ that for certain $f, g \in L^{2}\left(\mathbb{R}^{3} ; \mathbb{C}^{4}\right)$, the set function $\left(M_{t} f, g\right)$ may actually be the restriction to $\mathcal{S}_{t}$ of a scalar measure, we state the following simple consequence of the dominated convergence theorem and [K, VIII.1.6].

6.4 Proposition. Let $f, g \in L^{2}\left(\mathbb{R}^{3} ; \mathbb{C}^{4}\right)$ be functions for which $\left(M_{t} f, g\right)$ is the restriction to $\mathcal{S}_{t}$ of a scalar measure and let $V: \mathbb{R}^{3} \rightarrow \mathbb{R}$ be a spherically symmetric Borel measurable function in $L^{2}{ }_{\text {loc }}\left(\mathbb{R}^{3} \backslash\{0\}\right)$, such that for every $t>0$, the function $s \mapsto V(\omega(s)), 0 \leqq s \leqq t$ is integrable for $\left(M_{t} f, g\right)$-almost all $\omega \in \Omega$. Then for every $t>0$, the function $\omega \mapsto \exp \left[-i \int_{0}^{t} V(\omega(s)) d s\right], \omega \in \Omega$ is $\left(M_{t} f\right.$, g)-integrable.

Furthermore, if $D-V$ is essentially selfadjoint on $C_{c}^{\infty}\left(\mathbb{R}^{3}, \mathbb{C}^{4}\right)$, then

$$
\left(e^{i(D-V) t} f, g\right)=\int_{\Omega} \exp \left[-i \int_{0}^{t} V(\omega(s)) d s\right] d\left(M_{t} f, g\right)(\omega),
$$

for all $t>0$.

\section{References}

[A] Arai, M., On essential selfadjointness, distinguished selfadjoint extension and essential spectrum of Dirac operators with matrix valued potentials, Publ. RIMS, Kyoto Univ., 19 (1983), 33-57.

[B] Bartle, R., A general bilinear vector integral, Studia Math., 15 (1956), 337352.

[Be] Behncke, H., The Dirac equation with anomalous magnetic moment, Math. Z., 174 (1980), 213-225.

[Br] Brenner, P., The Cauchy problem for symmetric hyperbolic systems in $L_{p}$, Math. Scand., 19 (1966), 27-37.

[D-U] Diestel, J. and Uhl Jr., J.J., Vector Measures, Math. Surveys No. 15, Amer. Math. Soc., Providence, 1977.

[G-J] Glimm, J. and Jaffe, A., Quantum Physics: A Functional Integral Point of View, Springer-Verlag, New York, 1981.

[H-P] Hille, E. and Phillips, R.S., Functional Analysis and Semigroups, Amer. Math. Soc. Colloq. Publ. XXXI, New York, 1957.

[II] Ichinose, T., Path integral for the Dirac equation in two space-time dimensions, Proc. Japan Acad., 58A (1982), 290-293.

[12] - Path integral formulation of the propagator for a two dimensional Dirac particle, Physica 124A (1984), 419-426.

[I-T1] Ichinose, T. and Tamura, H., Propagation of a Dirac particle: a path integral approach, J. Math. Phys., 25 (1984), 1810-1819.

[I-T2] - Path integral approach to relativistic quantum mechanics: twodimensional Dirac equation, Prog. Theor. Phys. Suppl., 92 (1987), 144-175.

[I-T3] - Zitterbewegung of a Dirac particle in two-dimensional space-time, $J$. Math. Phys., 29 (1988), 103-109.

[J1] Jefferies, B., Integration with respect to closable set functions, J. Funct. Anal., 
66 (1986), 381-405.

[J2] , Remarks on the Feynman representation, Publ. RIMS, Kyoto Univ. 21 (1985), 1311-1323.

[J3] - Processes associated with evolution equations, J. Funct. Anal., 91 (1990), 259-277.

[J4] - An operator bound related to Feynman-Kac formulae, Proc. Amer. Math. Soc., in press.

[K] Kato, T., Perturbation Theory for Linear Operators, 2nd ed., Springer-Verlag, Berlin/New York, 1980.

[Klu] Kluvánek, I., Operator valued measures and perturbations of semi-groups, Arch. Rat. Mech. Anal., 81(2) (1983), 161-180.

[K-K] Kluvánek, I. and Knowles, G., Vector Measures and Control Systems, North Holland, Amsterdam, 1976.

[KS] Kalf, H., Schmincke, U.-W., Walter, J. and Wuist, R., On the spectral theory of Schrödinger and Dirac operators with strongly singular potentials, Lect. Note. in Math., 448 p. 182-226, Springer, Berlin-Heidelberg-New York, 1975.

[Ne] Neveu, J., Mathematical Foundations of the Calculus of Probability, HoldenDay, San Francisco/London, 1965.

[P1] Popov, V.S., On the properties of the discrete spectrum for $Z$ close to 137 , Soviet Physics JETP, 33(4) (1971), 665-673.

[P2] - "Collapse to the center" at $Z>137$ and critical nuclear charge, Soviet J. Nuclear Phys., 12(2) (1971), 235-243.

[R-S] Reed, M. and Simon, B., Methods of Modern Mathematical Physics, I, II, Academic Press, New York, 1975.

[S] Schwartz, L., Radon Measures in Arbitrary Topological Spaces, Tata Inst. Fund. Res., Oxford Univ. Press, 1973.

[Si] Simon, B., Functional Integration and Quantum Physics, Academic Press, New York/San Francisco/London, 1979.

[T」 Trotter, H.F., Approximation of semi-groups of operators, Pacific J. Math., 8 (1958), 887-919.

[W] Weidmann, J., Spectral Theory of Differential Operators, Lect. Note. in Math., 1258, Springer-Verlag, Berlin/Heidelberg/New York, 1987.

[Z] Zastawniak, T., Path integrals for the Dirac equation-some recent developments in the mathematical theory, Stochastic Analysis, Path Integration and Dynamics, K.D. Elworthy and J-C Zambrini, eds., Pitman Res. Notes in Math., 200, Longman Scientific and Technical, 1989. 
